\title{
PARAMETER SPACES FOR ALGEBRAIC EQUIVALENCE
}

\author{
JEFFREY D. ACHTER, SEBASTIAN CASALAINA-MARTIN, AND CHARLES VIAL
}

\begin{abstract}
A cycle is algebraically trivial if it can be exhibited as the difference of two fibers in a family of cycles parameterized by a smooth integral scheme. Over an algebraically closed field, it is a result of Weil that it suffices to consider families of cycles parameterized by curves, or by abelian varieties. In this paper, we extend these results to arbitrary base fields. The strengthening of these results turns out to be a key step in our work elsewhere extending Murre's results on algebraic representatives for varieties over algebraically closed fields to arbitrary perfect fields.
\end{abstract}

\section{INTRODUCTION}

Consider a scheme $X$ of finite type over a field $K$. Given a non-negative integer $i$, the group of dimension- $i$ cycles, denoted $\mathrm{Z}_{i}(X)$, is the free abelian group generated by closed integral subschemes of $X$ of dimension $i$. The Chow group $\mathrm{CH}_{i}(X)$ is the quotient of $\mathrm{Z}_{i}(X)$ by rational equivalence. A dimension- $i$ cycle class $a \in \mathrm{CH}_{i}(X)$ on $X$ is called algebraically trivial if there exist a twice- $K$-pointed smooth integral scheme $\left(T, t_{0}, t_{1}\right)$ of finite type over $K$, and a cycle class $Z \in \mathrm{CH}_{i+\operatorname{dim}_{K} T}\left(T \times_{K} X\right)$, such that $a=Z_{t_{1}}-Z_{t_{0}}$ in $\mathrm{CH}_{i}(X)$. Here $Z_{t_{j}}, j=0,1$, is the refined Gysin fiber of the cycle class $Z$ [Ful98, §6.2]. Algebraic equivalence defines an equivalence relation on $Z_{i}(X)$; in fact algebraic equivalence is an adequate equivalence relation (e.g., [Sam60], [Ful98, $\S 10.3$, Prop. 10.3], [Mur85, §1.1]).

Over an algebraically closed field $K=\bar{K}$, Weil showed ([Wei54, Lem. 9] ; see also [Lan59, §III.1], [Sam60], [Ful98, Exa. 10.3.2]) that if one insists further in the definition of algebraic equivalence that the parameter space $T$ be a smooth projective curve, or an abelian variety, one arrives at the same equivalence relation on $\mathrm{CH}_{i}(X)$. The first goal of the present paper is to secure these results in the case where the base field is not assumed to be algebraically closed.

Theorem 1 (Proposition 3.14). Let $X$ be a scheme of finite type over a perfect field $K$, and let $a \in \mathrm{CH}_{i}(X)$ be an algebraically trivial cycle class. Then there exist a smooth projective integral curve (resp. abelian variety) $T$ over $K$, a cycle class $Z \in \mathrm{CH}_{i+\operatorname{dim} T}\left(T \times_{K} X\right)$, and a pair of $K$-points $t_{0}, t_{1} \in T(K)$, such that $a=Z_{t_{1}}-Z_{t_{0}}$ in $\mathrm{CH}_{i}\left(X_{K}\right)$.

We also consider a notion of algebraic triviality for cycles defined via flat families (see §3.6) and we show that algebraic triviality in this sense is the same as algebraic triviality as defined in [Ful98, Def. 10.3]. If in Theorem 1 the field $K$ is taken to be imperfect of characteristic $p$, we obtain the same result taking cycles with $\mathbb{Z}[1 / p]$-coefficients ; see Proposition 4.10 and Theorem 4.11 , as well as Proposition 3.14 for a related result.

In this paper we are also interested in a slightly more general question. Let $L / K$ be a separable algebraic extension of fields and assume that $a \in \mathrm{CH}_{i}\left(X_{L}\right)$ is an algebraically trivial class on

Date: October 17, 2019.

MSC2010 classification: 14C25, 14C05, 14C15, 14G27, 14K99.

Key words: Algebraic cycles, algebraic equivalence, abelian varieties.

The first author was partially supported by grants from the Simons Foundation (204164) and the NSA (H9823014-1-0161, H98230-15-1-0247 and H98230-16-1-0046). The second author was partially supported by NSF grant DMS-1101333, a Simons Foundation Collaboration Grant for Mathematicians (317572), and NSA grant H98230-161-0053. The third author was supported by EPSRC Early Career Fellowship EP/K005545/1. 
$X_{L}:=X \times_{K} L$. Theorem 1 shows that there exist a smooth integral scheme $T$ of finite type over $L$, a cycle class $Z \in \mathrm{CH}_{i+\operatorname{dim} T}\left(T \times_{L} X_{L}\right)$, and a pair of $L$-points $t_{0}, t_{1} \in T(L)$, such that $a=Z_{t_{1}}-Z_{t_{0}}$ in $\mathrm{CH}_{i}\left(X_{L}\right)$, where $T$ may be taken to be a smooth projective curve, or an abelian variety. We show that in this situation, one may in fact take $T$ and $Z$ to be defined over $K$, with $T$ geometrically integral. This technical strengthening of Theorem 1 turns out to be a key step in extending Murre's work [Mur85] on algebraic representatives for varieties over algebraically closed fields to arbitrary perfect fields; see [ACMV17] for details, and specifically Lemma 4.9 and Corollary 4.10 therein.

Theorem 2 (Theorem 4.11, Proposition 4.10). Let $X$ be a scheme of finite type over $K$, let $L / K$ be a separable algebraic extension of fields, and let $a \in \mathrm{CH}_{i}\left(X_{L}\right)$ be an algebraically trivial cycle class. Then there exist a smooth geometrically integral quasi-projective curve of finite type $T$ over $K, a$ cycle class $Z \in \mathrm{CH}_{i+\operatorname{dim} T}\left(T \times_{K} X\right)$, and a pair of L-points $t_{0}, t_{1} \in T(L)$, such that $a=Z_{t_{1}}-Z_{t_{0}}$ in $\mathrm{CH}_{i}\left(X_{L}\right)$. Moreover, if $K$ is perfect then $T$ may be taken to be a smooth geometrically integral projective curve, or an abelian variety.

Clearly Theorem 1 follows from Theorem 2, as the special case where $L=K$. If in the last assertion of Theorem 2 the field $K$ is taken to be imperfect of characteristic $p$, we obtain the same result using cycles with $\mathbb{Z}[1 / p]$-coefficients; see Proposition 4.10 and Theorem 4.11. Aspects of the proof of the theorem are inspired by the arguments of Weil. Consequently, in the process of proving the result, we also provide a modern treatment of some of those arguments. However, there are several places where our treatment diverges significantly from Weil. In short, using the refined Gysin homomorphism on cycle classes from [Ful98], we are able to develop arguments without the need for a base change of field. The first place this is apparent is when we allow for taking fibers of cycle classes over 0-cycles in the parameter space (see §3.4). This makes it relatively easy to show that one may use curves as the parameter spaces for algebraic triviality, without a base change of fields. The second place is in showing that one may move from curves as parameter spaces to abelian varieties as parameter spaces. Here, the refined Gysin homomorphism allows us to use less Brill-Noether theory, and in particular, to work without the base change of field in Weil's arguments. For comparison, we include a modern treatment of Weil's argument in the appendix, to emphasize where this crucial part of our argument differs from Weil. Finally, to prove Theorem 2, there are several new ingredients concerning moving from algebraic triviality over $L$, to questions about cycles over $K$ (see especially Lemma 4.14). Our results in fact give something stronger than Theorem 2 ; in particular, we investigate some related notions of algebraic triviality that are motivated by the notion of $\tau$-algebraic equivalence of [Kle68]. We direct the reader to Theorem 4.11 for precise statements.

Acknowledgments. We thank the referee for helpful suggestions.

Notation and conventions. We follow the conventions of [Fu198] for the set-up of algebraic cycles on schemes over fields, the formalism of intersection theory, and the definitions of various notions of equivalence of cycles. In particular, following [Ful98], we define a variety over a field $K$ to be an integral scheme of finite type over $K$. A curve over $K$ is a quasi-projective variety of dimension 1 over $K$. The dimension of an irreducible scheme of finite type $T$ over $K$ will be denoted $d_{T}$. Given a commutative ring with unit $R$, by $\mathrm{CH}_{i}(-)_{R}$ we mean $\mathrm{CH}_{i}(-) \otimes_{\mathbb{Z}} R$. The symbol $\bar{K}$ denotes an algebraic closure of the field $K$.

We also fix some notation for closed points of schemes. Let $X$ be a scheme of finite type over a field $K$. A closed point of $X$ is an element $P$ of the topological space $|X|$ such that $\{P\}$ is closed. Associated to a closed point $P$ of $X$ is a 0 -dimensional closed subscheme $\operatorname{Spec} \kappa(P) \rightarrow X$, where $\kappa(P)$ is the residue field at $P$. We will denote this closed subscheme of $X$ by using square brackets; e.g., $[P]$. We will denote the 0 -cycle (and cycle class) associated to $P$ also by $[P]$. A point $P \in|X|$ is closed if and only if the residue field $\kappa(P)$ is a finite extension of $K$. If $L$ is a field, and 
$p:$ Spec $L \rightarrow X$ is an $L$-point of $X$, we will typically denote the image of $p$ with the corresponding upper case letter; e.g., $P \in|X|$. The image point $P$ is closed if $L / K$ is algebraic.

\section{Symmetric PRODUCTS OF SCHEMES}

This section contains some preliminaries on symmetric products of schemes, which will be used in Section 3.4. We expect the content of this section is well known to the experts, and our primary aim is to fix notation that will be used later.

For a scheme $T$, let $\Pi_{0}(T)$ denote its set of irreducible components. For a positive integer $N$, let $S^{N}(T)$ be the $N$-th symmetric power of $T$.

Lemma 2.1. Let $T$ be an integral scheme of finite type over a field $K$, and let $e=\# \Pi_{0}\left(T_{\bar{K}}\right)$. For each positive integer $d$, let

$$
S^{\Delta_{d}}\left(T_{\bar{K}}\right)=\prod_{\bar{D} \in \Pi_{0}\left(T_{\bar{K}}\right)} S^{d}(\bar{D}) \subset S^{d e}\left(T_{\bar{K}}\right) .
$$

(1) Then $S^{\Delta_{d}}\left(T_{\bar{K}}\right)$ descends to a geometrically irreducible component $S^{\Delta_{d}}(T)$ of $S^{\text {de }}(T)$.

(2) The natural map $S^{d e}(T) \times S^{f e}(T) \rightarrow S^{(d+f) e}(T)$ restricts to $S^{\Delta_{d}}(T) \times S^{\Delta_{f}}(T) \rightarrow S^{\Delta_{d+f}}(T)$.

Proof. Fix a component $\bar{D} \in \Pi_{0}\left(T_{\bar{K}}\right)$, and let $H \subset \operatorname{Gal}(K)$ be its stabilizer. Since $T$ is irreducible, we have

$$
T_{\bar{K}}=\bigsqcup_{[\sigma] \in \operatorname{Gal}(K) / H} \bar{D}^{\sigma}
$$

Let $e=\# \Pi_{0}\left(T_{\bar{K}}\right)$. Inside the de-th symmetric power $S^{d e}(T)_{\bar{K}}=S^{d e}\left(T_{\bar{K}}\right)$ we identify the irreducible component

$$
S^{\Delta_{d}}\left(T_{\bar{K}}\right)=\prod_{[\sigma] \in \operatorname{Gal}(K) / H} S^{d}\left(\bar{D}^{\sigma}\right) .
$$

Since this element of $\Pi_{0}\left(S^{\text {de }}\left(T_{\bar{K}}\right)\right.$ ) is fixed by $\operatorname{Gal}(K)$ (and since all geometrically irreducible components of a scheme over $K$ are defined over a separable closure of $K$ ), it descends to $K$ as a geometrically irreducible scheme. Part (2) is obvious.

Lemma 2.2. Let $T / K$ be a normal integral scheme of finite type over a field $K$, and let $e=$ $\# \Pi_{0}\left(T_{\bar{K}}\right)$. An effective zero-cycle of degree d determines a $K$-point of $S^{\Delta_{d / e}}(T)$.

Proof. By Lemma 2.1(2), it is enough to consider the case of closed points on $T$. It is clear that a closed point $P \rightarrow T$ of degree $d$ determines a $K$-point of $S^{d}(T)$; the content of the assertion is that this point lies in $S^{\Delta_{d / e}}(T)$. If the field of definition of the components of $T_{\bar{K}}$ is Galois over $K$, then the statement is easy. In general, we need to work a little harder.

Let $K_{c}$ be the separable algebraic closure of $K$ inside $K(T)$. Then $T$ is a geometrically irreducible scheme of finite type over $K_{c}$. Now let $P$ be a closed point of $T$ with residue field $L=\kappa(P)$, and let Spec $A$ be an open affine subscheme of $T$ which contains $P$; let $\mathfrak{p} \subset A$ be the corresponding ideal. The natural maps $K \hookrightarrow A \rightarrow L$ yield an inclusion $K_{c} \hookrightarrow L$; then $K_{c}$ is contained in $L_{s}$, the separable closure of $K$ inside $L$.

The canonical surjection $\pi: A \rightarrow A / \mathfrak{p}$ yields, after base change, a surjection $\pi_{\bar{K}}: A \otimes_{K} \bar{K} \rightarrow$ $A / \mathfrak{p} \otimes_{K} \bar{K}$. On one hand, irreducible components of $\operatorname{Spec}\left(A \otimes_{K} \bar{K}\right)$, and thus of $T_{\bar{K}}$, are in bijection with minimal ideals of $A \otimes_{K} \bar{K}$. Similarly, the irreducible components of $P_{\bar{K}}=\operatorname{Spec}\left(A / \mathfrak{p} \otimes_{K} \bar{K}\right)-$ that is, the points of $P_{\bar{K}}$ lying over $P$ - are in bijection with minimal ideals of $(A / \mathfrak{p}) \otimes_{K} \bar{K}$. If $\mathfrak{q}$ is a minimal prime of $(A / \mathfrak{p}) \otimes_{K} \bar{K}$, then the component of $T_{\bar{K}}$ containing the corresponding point is indexed by the minimal prime ideal of $\pi_{\bar{K}}^{-1}(\mathfrak{q})$. 
On the other hand, irreducible components of $\operatorname{Spec}\left(A \otimes_{K} \bar{K}\right)$ are also indexed by $\operatorname{Hom}_{K}\left(K_{c}, \bar{K}\right)$, insofar as

$$
A \otimes_{K} \bar{K} \cong \oplus_{\sigma \in \operatorname{Hom}_{K}\left(K_{c}, \bar{K}\right)} A \otimes_{K_{c}, \sigma} \bar{K}
$$

the irreducible component indexed by $\sigma$ corresponds to the minimal prime ideal containing $\operatorname{ker}\left(A \otimes_{K}\right.$ $\left.\bar{K} \rightarrow A \otimes_{K_{c}, \sigma} \bar{K}\right)$.

Similarly, irreducible components of $P_{\bar{K}}$ are indexed by $\operatorname{Hom}_{K}(L, \bar{K})$. Moreover, we have

$$
\begin{aligned}
(A / \mathfrak{p}) \otimes_{K} \bar{K} & \cong \oplus_{\sigma \in \operatorname{Hom}_{K}\left(K_{c}, \bar{K}\right)}\left(L \otimes_{K_{c}, \sigma} \bar{K}\right) \\
& \cong \oplus_{\sigma} \oplus_{\tau \in \operatorname{Hom}_{K_{C}}(L, \bar{K}):\left.\tau\right|_{K_{c}}=\sigma} L \otimes_{\tau, K_{c}, \sigma} \bar{K} .
\end{aligned}
$$

Comparison of minimal primes of $A \otimes_{K} \bar{K}$ and $(A / \mathfrak{p}) \otimes_{K} \bar{K}$ then shows that if $Q \in \Pi_{0}\left(P_{\bar{K}}\right)$ corresponds to $\tau \in \operatorname{Hom}_{K}(L, \bar{K})$, then the irreducible component of $\operatorname{Spec}\left(A \otimes_{K} \bar{K}\right)$ supporting it corresponds to $\left.\tau\right|_{K_{c}}$.

Since, for a given $\sigma \in \operatorname{Hom}_{K}\left(K_{c}, \bar{K}\right)$,

$$
\#\left\{\tau \in \operatorname{Hom}_{K}(L, \bar{K}):\left.\tau\right|_{K_{c}}=\sigma\right\}=\#\left\{\tau \in \operatorname{Hom}_{K}\left(L_{s}, \bar{K}\right):\left.\tau\right|_{K_{c}}=\sigma\right\}=\left[L_{s}: K_{c}\right]
$$

is independent of $\sigma$, points of $P_{\bar{K}}$ are uniformly distributed among the components of $T_{\bar{K}}$.

\section{Algebraic equivalence: the Classical Story}

In this section, we recall various definitions of algebraic equivalence for cycles classes. These depend on constraints imposed on the smooth parameter spaces. A classical result of Weil [Wei54] says that these definitions all agree when working over an algebraically closed ground field. We show in Proposition 3.14 that this holds over an arbitrary perfect ground field. We also recall a definition of flat algebraic equivalence for cycles (as opposed to cycle classes), show it is equivalent to the definition of algebraic equivalence of cycles in [Fu198, Def. 10.3], and recall how it can be used to reformulate rational triviality. We close this section by showing that, in a suitable sense, algebraic triviality of cycles on $X$ is independent of the choice of base field.

In this section, we fix a field $K$ and work in the category of schemes of finite type over $K$.

3.1. Definition of algebraically trivial cycle classes. Let $X$ be a scheme of finite type over some field $K$. Recall from [Ful98, §1.3] that a cycle $\alpha \in \mathrm{Z}_{i}(X)$ is said to be rationally equivalent to zero if there are a finite number of $(i+1)$-dimensional closed integral sub-schemes $W_{r}$ of $X$, and non-zero rational functions $f_{r}$ in the function field of $W_{r}$, such that $\alpha$ is the sum of the cycles associated to $\operatorname{div}\left(f_{r}\right)$. In particular, rational equivalence does not depend on the choice of a base field over which $X$ is of finite type. The collection of $i$-dimensional cycles that are rationally equivalent to zero form a subgroup of the group of $i$-dimensional cycles $\mathrm{Z}_{i}(X)$, and the Chow group of $i$-dimensional cycle classes is the quotient of $\mathrm{Z}_{i}(X)$ by this subgroup. The cycle class associated to a cycle is the image of that cycle in the Chow group.

Definition 3.1 (Algebraically trivial cycle class [Fu198, Def. 10.3]). Let $X$ be a scheme of finite type over a field $K$. A dimension- $i$ cycle class $a \in \mathrm{CH}_{i}(X)$ on $X$ is called algebraically trivial if there exist a smooth integral scheme $T$ of finite type over $K$, an $\left(i+d_{T}\right)$-dimensional cycle class $Z \in \mathrm{CH}_{i+d_{T}}\left(T \times_{K} X\right)$, and $K$-points $t_{1}, t_{0} \in T(K)$ such that $Z_{t_{1}}-Z_{t_{0}}=a$ in $\mathrm{CH}_{i}(X)$. Here $Z_{t_{j}}$, $j=0,1$, is the refined Gysin fiber of the cycle class $Z$ [Ful98, $\S 6.2]$.

The subset of $\mathrm{CH}_{i}(X)$ consisting of algebraically trivial cycle classes forms a group ; it thus makes sense to define a dimension- $i$ cycle class with $R$-coefficients $a \in \mathrm{CH}_{i}(X)_{R}$ to be algebraically trivial if it is an $R$-linear combination of algebraically trivial cycles in the sense of Definition 3.1. 
For schemes of finite type over $K$, this group is stable under the usual operations of proper pushforward, flat pull-back, refined Gysin homomorphisms, and Chern class operations; see [Ful98, Prop. 10.3] and Proposition 3.4 below.

Remark 3.2. Several authors require the parameter space $T$ in Definition 3.1 to be separated, or even quasi-projective. For instance, Kleiman [Kle68] and Jannsen [Jan00] require the parameter space to be quasi-projective (in fact the parameter spaces are taken to be projective in [Jan00], but over a perfect field, by Bertini's theorem and Lemma 3.8, below, this is equivalent to quasiprojective), and Hartshorne [Har73] seems to require the parameter space to be separated but works over an algebraically closed field. Note that Fulton [Ful98] defines a variety as an integral scheme of finite type over a field, and thus does not require the parameter space to be separated (cf. [Ful98, B.2.3]) ; we have followed this more general convention. As we will recall in Proposition 3.14, these conventions all lead to the same notion of triviality over a perfect field.

Remark 3.3. In Definition 3.1 the parameter scheme $T$ is always geometrically integral. This is because a smooth integral scheme of finite type over a field $K$ that admits a $K$-point is geometrically integral.

3.2. Related notions of triviality for cycle classes. There are other notions of triviality used in the literature that are obtained by imposing constraints on the parameter space. They are all equivalent over an algebraically closed field by Weil [Wei54, Lem. 9] ; our main result, Theorem 1 (Proposition 3.14), extends Weil's result by showing that they are all equivalent over a perfect field.

Definition 3.4 (Parameter space algebraically trivial cycle classes). Let $X$ be a scheme of finite type over a field $K$. Let $a$ be a cycle class in $\mathrm{CH}_{i}(X)$. Suppose there exist a smooth integral scheme $T$ of finite type over $K$, a cycle class $Z \in \mathrm{CH}_{i+d_{T}}\left(T \times_{K} X\right)$, and $K$-points $t_{1}, t_{0} \in T(K)$ such that $Z_{t_{1}}-Z_{t_{0}}=a$ in $\mathrm{CH}_{i}(X)$. If $T$ can be taken to be a curve (resp. abelian variety, resp. projective curve, resp. quasi-projective scheme, resp. separated scheme), then we say that $a$ is curve (resp. abelian variety, resp. projective curve, resp. quasi-projective scheme, resp. separated scheme) trivial.

Proposition 3.5 ([Ful98, Prop. 10.3]). For any of the notions of triviality in Definition 3.4 above, the trivial cycles classes form a subgroup of the group of all cycle classes. This subgroup is preserved by the basic operations:

(1) proper push-forward;

(2) flat pull-back;

(3) refined Gysin homomorphisms;

(4) Chern class operations.

Proof. The proof is the same as [Ful98, Prop. 10.3]. The classes of abelian varieties, quasi-projective schemes, and projective schemes are each stable under products; in the case of (projective) curves, we in addition use Bertini's theorem (Theorem A.1) to cut the product of (projective) curves back down to a (projective) curve. That the four operations (1)-(4) are preserved follows from the corresponding parts (a)-(d) of [Ful98, Prop. 10.1].

Remark 3.6. By virtue of the previous proposition, given a commutative ring $R$, we can extend the notions of triviality in Definitions 3.1 and 3.4 to Chow groups with $R$-coefficients by extending $R$-linearly. For instance, $a \in \mathrm{CH}^{i}(X)_{R}$ is said to be curve trivial if it is an $R$-linear combination of curve trivial classes in $\mathrm{CH}_{i}(X)$ (as in Definition 3.4). All of the results in Section 3 hold in this more general setting.

Remark 3.7. We have the following elementary implications among the notions of triviality:

ab. var. triv. $\Longrightarrow$ proj. curve triv. $\Longrightarrow$ curve triv. $\Longleftrightarrow$ q.p. sch. triv. $\Longrightarrow$ sep. sch. triv. $\Longrightarrow$ alg. triv. 
Indeed, all the implications are immediate from the definitions, except for the implications "Abelian variety trivial $\Longrightarrow$ Projective curve trivial" and "Quasi-projective scheme trivial $\Longrightarrow$ Curve trivial". These follow from applying Bertini's theorem (Theorem A.1).

3.3. Projective curve triviality. Since every smooth integral curve over a field has a regular projective model (the normalization of any projective completion), over a perfect field we have "Projective curve trivial" $\Longleftrightarrow$ "Curve trivial". However, over an imperfect field, there exist projective curves that are regular but not smooth. Such a curve admits no surjective morphism from a smooth projective scheme (this follows from Bertini's theorem and [Sta17, Tag 0CCW]); in particular, it admits no smooth projective model. Thus the reverse implication over imperfect fields is less clear. The following lemma addresses this:

Lemma 3.8. Let $X / K$ be a scheme of finite type, and let $a \in \mathrm{CH}_{i}(X)$ be a curve trivial cycle class.

(1) If $K$ is perfect, then a is projective curve trivial.

(2) If $K$ is imperfect of characteristic $p$, then there exists some $n$ such that $p^{n}$ a is projective curve trivial.

Proof. Suppose $T$ is a smooth curve and $Z, t_{1}$ and $t_{0}$ are as in Definition 3.4. Let $\widetilde{T}$ be the regular projective model of $T$, and let $Z^{\prime}$ be a pre-image of the cycle class $Z$ under the surjective [Ful98, Prop. 1.8] restriction map $\mathrm{CH}_{i+d_{T}}\left(\widetilde{T} \times_{K} X\right) \rightarrow \mathrm{CH}_{i+d_{T}}\left(T \times_{K} X\right)$. Then $Z_{t_{1}}^{\prime}-Z_{t_{0}}^{\prime}=Z_{t_{1}}-Z_{t_{0}}$.

First, suppose $K$ is perfect. Then $\widetilde{T}$ is smooth, and we are done.

Second, suppose $K$ is imperfect of characteristic $p$. Let $\widetilde{T}^{\left(p^{n}\right)}$ denote the pullback of $\widetilde{T}$ by the iterated Frobenius map $\lambda \mapsto \lambda^{p^{n}}$ of $K$. The iterated relative Frobenius map $F_{\widetilde{T} / K}^{n}: \widetilde{T} \rightarrow \widetilde{T}^{\left(p^{n}\right)}$ is finite and flat of degree $p^{n}$, and for sufficiently large $n$ the normalization $W_{n}$ of $\widetilde{T}^{\left(p^{n}\right)}$ is smooth [Sch09, Lem. 1.2]. Fix one such $n$, and let $\nu: W_{n} \rightarrow \widetilde{T}^{\left(p^{n}\right)}$ be the normalization map. Since $\widetilde{T}$ is smooth at each $K$-point $t_{i}$, there are unique $K$-points $w_{i}$ of $W_{n}$ above each $F_{\widetilde{T} / K}^{n}\left(t_{i}\right)$. Let $Y=\nu^{*} F_{\widetilde{T} / K}^{n}\left(Z^{\prime}\right)$. Then $Y_{w_{1}}-Y_{w_{0}}=p^{n}\left(Z_{w_{1}}-Z_{w_{0}}\right)$.

3.4. Algebraically trivial cycles using 0-cycles of degree 0. Algebraic equivalence of cycle classes is defined by using the difference of two Gysin fibers over two $K$-points of the parameter scheme. Working more generally with 0 -cycles of degree 0 on the parameter scheme provides more flexibility. Lemma 3.9 and Proposition 3.10 below say that by doing so we in fact obtain the same equivalence relation on cycles. In addition, Proposition 3.10 establishes that algebraic triviality coincides with quasi-projective scheme triviality.

Let $P_{1}, \ldots, P_{r}$ be closed points of a scheme $X$ of finite type over a field $K$, with residue fields $\kappa\left(P_{i}\right)$, respectively. By definition, the degree of the zero-cycle $\beta=\sum_{i} n_{i}\left[P_{i}\right] \in \mathrm{Z}_{0}(X)$ is the integer $\operatorname{deg} \beta:=\sum_{i} n_{i}\left[\kappa\left(P_{i}\right): K\right]$. If $X$ is proper over $K$, this agrees with the definition via proper pushforward to Spec $K$ [Ful98, Def. 1.4]. Given a smooth scheme $T$ of finite type over $K$, a cycle class $Z \in \mathrm{CH}_{i+d_{T}}\left(T \times_{K} X\right)$, and a zero-cycle $\beta=\sum_{i} n_{i}\left[P_{i}\right] \in \mathrm{Z}_{0}(T)$, we define

$$
Z_{\beta}:=\sum_{i} n_{i} Z_{\left[P_{i}\right]} \in \mathrm{CH}_{i}(X)
$$

where $Z_{\left[P_{i}\right]}$ denotes the image of the refined Gysin fiber of $Z$ over the closed subscheme $\left[P_{i}\right]$ of $T$ (which is regularly embedded since $T$ is smooth) pushed forward along the projection $\operatorname{Spec} \kappa\left(P_{i}\right) \times_{T}$ $\left(T \times_{K} X\right)=: X_{\left[P_{i}\right]} \rightarrow X$; this is also the finite morphism obtained by pulling back $X$ along the finite morphism $\operatorname{Spec} \kappa\left(P_{i}\right) \rightarrow \operatorname{Spec} K$.

Lemma 3.9. Let $X$ be a scheme of finite type over a field $K$. Let $T$ be a smooth integral quasiprojective scheme over $K$ and let $\beta \in \mathrm{Z}_{0}(T)$ with $\operatorname{deg} \beta=0$. Let $Z \in \mathrm{CH}_{i+d_{T}}\left(T \times_{K} X\right)$ be a cycle class on $T \times_{K} X$. Then the cycle class $Z_{\beta} \in \mathrm{CH}_{i}(X)$ is quasi-projective scheme trivial. 
Proof. Applying Bertini's theorem (Theorem A.1) gives a smooth integral quasi-projective curve $g: C \hookrightarrow T$ over $K$, passing through the support of the zero-cycle $\beta$. Denote the corresponding zero-cycle of $C$ by $\gamma$. For the cycle $Z^{\prime}:=g^{!} V \in \mathrm{CH}_{i+1}\left(C \times_{K} X\right)$, we have $Z_{\gamma}^{\prime}=Z_{\beta}$ in $\mathrm{CH}_{i}(X)$ ([Ful98, Thm. 6.5]). Thus we may and do assume that $T$ is a smooth integral quasi-projective curve.

Recall that for any positive integer $N$, we let $S^{N} T$ denote the $N$-th symmetric power of $T$. Since $T$ is a smooth integral quasi-projective curve, $S^{N} T$ is a smooth integral quasi-projective scheme. Let $S^{N} Z$ be the induced cycle on $S^{N} T \times_{K} X$. More precisely, view $S^{N} T$ as a component of the Hilbert scheme of $T$ (see e.g., [Kle05, Thm. 9.3.7, Rem. 9.3.9]), and let $\mathcal{D} \subseteq S^{N} T \times_{K} T$ be the universal divisor; $\mathcal{D}:=\{(z, x): x \in \operatorname{Supp} z\}$. Let $p: S^{N} T \times_{K} T \rightarrow S^{N} T$ and $q: S^{N} T \times_{K} T \rightarrow T$ be the natural projections. Then we can define

$$
S^{N} Z:=\left(\left.p\right|_{\mathcal{D}} \times \operatorname{id}_{X}\right)_{*}\left(\left.q\right|_{\mathcal{D}} \times \operatorname{id}_{X}\right)^{*} Z \in \mathrm{CH}_{i+N}\left(S^{N} T \times_{K} X\right)
$$

where $\left(\left.q\right|_{\mathcal{D}} \times \operatorname{id}_{X}\right)^{*}$ is the flat pull-back ( $\mathcal{D}$ is smooth and integral, so that the dominant morphism $\left.q\right|_{\mathcal{D}}: \mathcal{D} \rightarrow T$ is flat $)$ and $\left(\left.p\right|_{\mathcal{D}} \times \mathrm{id}_{X}\right)_{*}$ is the proper push-forward $\left(\left.p\right|_{\mathcal{D}}: \mathcal{D} \rightarrow S^{N} T\right.$ is finite, in particular proper). The fiber of $S^{N} Z$ over $r_{1}+\cdots+r_{N} \in S^{N} T$ is then $\sum Z_{r_{i}}$.

Note that an effective zero-cycle of degree $N$ on $T$ determines a rational $K$-point of $S^{N} T$. The key point now is that, even though the smooth integral curve $T$ over $K$ may not have a $K$-point and may be geometrically reducible, there is an irreducible component of a symmetric product of $T$ that has a $K$-point and is geometrically irreducible.

If $T_{\bar{K}}$ has exactly $e$ irreducible components, then for each positive integer $d$ we have a geometrically irreducible component (Lemma 2.1)

$$
S^{\Delta_{d}}(T) \subset S^{d e}(T)
$$

of the symmetric product, parameterizing those de-tuples with equal weight in each geometric component of $T_{\bar{K}}$. The degree of any zero-cycle on $T$ is a multiple of $e$, and an effective zero-cycle of degree de on $T$ determines then a rational $K$-point $P \rightarrow S^{\Delta_{d}} T$ (Lemma 2.2).

Let us then write $\beta=\sum_{i=1}^{r} n_{i}\left[Q_{i}\right]-\sum_{j=1}^{s} m_{j}\left[R_{j}\right]$, where $n_{i}>0$ and $m_{j}>0$ for all $i, j$. Let $N:=$ $\sum_{i=1}^{r} n_{i}\left[\kappa\left(Q_{i}\right): K\right]=\sum_{j=1}^{s} m_{j}\left[\kappa\left(R_{j}\right): K\right]$, and consider $t_{0}:=\sum_{i=1}^{r} n_{i} Q_{i}$ and $t_{1}:=\sum_{j=1}^{s} m_{j} R_{j}$ viewed as $K$-points of $S^{\Delta_{N / e}} T$. Then we find that $Z_{\beta}=\left(S^{\Delta_{N / e}} Z\right)_{t_{0}}-\left(S^{\Delta_{N / e}} Z\right)_{t_{1}}$, where $S^{\Delta_{N / e}} Z$ denotes the component of $S^{N} Z$ over $S^{\Delta_{N / e}} T \times X$. In other words, $Z_{\beta}$ is quasi-projective scheme trivial.

A corollary is the following further implication that can be added to the diagram of Remark 3.7 :

Proposition 3.10. Let $X$ be a scheme of finite type over a field $K$, and let $a \in \mathrm{CH}_{i}(X)$ be an algebraically trivial cycle class. Then a is quasi-projective scheme trivial. Thus, given Remark 3.7, we have the following implications among the notions of triviality:

ab. var. triv. $\Longrightarrow$ proj. curve triv. $\Longrightarrow$ curve triv. $\Leftrightarrow$ q.p. sch. triv. $\Leftrightarrow$ sep. sch. triv. $\Leftrightarrow$ alg. triv.

Proof. This is motivated by [Ful98, Exa. 10.3.2]. Let $X$ be a scheme of finite type over $K$, and let $a \in \mathrm{CH}_{i}(X)$ be an algebraically trivial cycle class. From the definition, there exist a smooth integral scheme $T$ of finite type over $K$, a pair of $K$-points $t_{1}, t_{0} \in T(K)$, and a cycle class $Z^{\prime} \in$ $\mathrm{CH}_{i+d_{T}}\left(T \times_{K} X\right)$, such that $a=Z_{t_{1}}^{\prime}-Z_{t_{0}}^{\prime}$. As mentioned in Remark 3.3, $T$ is geometrically integral, since it admits $K$-points.

By Bertini's theorem (Corollary A.3), there are smooth geometrically integral quasi-projective curves $C_{1}, \ldots, C_{r} \subseteq T$ over $K$ for some $r$, and closed points $T_{j_{1}}, T_{j_{0}}$ in $C_{j}$ for $j=1, \ldots, r$, such that $T_{j_{1}}=T_{(j+1)_{0}}$ for $j=1, \ldots, r-1$, and $T_{1_{0}}$ is the closed point associated to $t_{0}$, and $T_{r_{1}}$ is the closed point associated to $t_{1}$. Then if we let $Z^{(j)} \in \mathrm{CH}_{i+1}\left(C_{j} \times_{K} X\right)$ be the refined Gysin restriction of 
$Z^{\prime}$ to $C_{j}, j=1, \ldots, r$, we have

$$
a=\left(Z_{t_{1}}^{\prime}-Z_{t_{0}}^{\prime}\right)=\sum_{j=1}^{r}\left(Z^{(j)}\right)_{\left[T_{j_{1}}\right]}-\left(Z^{(j)}\right)_{\left[T_{j_{0}}\right]} \in \mathrm{CH}_{i}(X) .
$$

Here, as defined above in (3.1), the notation $\left(Z^{(j)}\right)_{\left[T_{j_{\ell}}\right]}$ indicates the push forward under the finite morphism $X_{\left[T_{j_{\ell}}\right]} \rightarrow X$ of the refined Gysin fiber of $Z^{(j)}$ in $\mathrm{CH}_{i}\left(X_{\left[T_{j_{\ell}}\right]}\right)$.

With this set-up, we now fix:

$$
\begin{aligned}
S & =C_{1} \times_{K} \cdots \times_{K} C_{r}, \quad \operatorname{pr}_{j}: S \rightarrow C_{j} \text { the } j \text {-th projection, } \\
s_{\ell} & =\left[T_{1_{\ell}}\right] \times_{K} \cdots \times_{K}\left[T_{r_{\ell}}\right] \in \mathrm{CH}_{0}\left(C_{1} \times_{K} \cdots \times_{K} C_{r}\right), \quad \ell=0,1, \\
V_{j} & =\operatorname{pr}_{j}^{*} Z^{(j)} \in \mathrm{CH}_{i+r}\left(S \times_{K} X\right), \quad j=1, \ldots, r \\
V & =\sum_{j=1}^{r} V_{j} \in \mathrm{CH}_{i+r}\left(S \times_{K} X\right) .
\end{aligned}
$$

The scheme $S$ is a smooth geometrically integral quasi-projective variety over $K$, and $s_{1}$ and $s_{0}$ are closed 0-dimensional subschemes of $S$; we have that $V_{s_{1}}-V_{s_{0}}=Z_{t_{1}}-Z_{t_{0}}=a$. Since $\operatorname{deg} s_{0}=\operatorname{deg} s_{1}$, we can apply Lemma 3.9 to conclude that $a$ is quasi-projective scheme trivial.

A zero-cycle $\beta$ is said to be numerically trivial if $\operatorname{deg} \beta=0$. The following proposition, which is a corollary of Lemma 3.9, is certainly well-known (e.g., [Ful98, 19.3.5, p.386] in the case $K=\mathbb{C}$ ) :

Proposition 3.11. Numerical and algebraic triviality agree for 0-cycles on smooth integral projective schemes over $K$.

Proof. If $X$ is a smooth geometrically integral quasi-projective scheme over $K$, we apply Lemma 3.9 to $T=X$ and $Z=\Delta_{X}$, the diagonal sitting in $X \times_{K} X$, and we find that any zero-cycle of degree 0 is algebraically trivial. Conversely, assuming furthermore that $X$ is proper, an algebraically trivial zero-cycle is numerically trivial by the principle of conservation of number [Ful98, §10.2].

Remark 3.12. The proposition is not true without the smoothness hypothesis (see Example 3.13) or the hypothesis that $X$ be proper (e.g., $X=\mathbb{A}_{K}^{1}$, where all 0-cycles are algebraically trivial).

Example 3.13. Consider the rational nodal curve $X=\left\{z y^{2}+z x^{2}-x^{3}=0\right\} \subseteq \mathbb{P}_{\mathbb{Q}}^{2}$ defined over $\mathbb{Q}$. Let $N=[0: 0: 1]$ be the node, and let $P=[1: 0: 1]$. One can check that $\mathrm{Z}_{0}(X) /($ algebraic equivalence $) \cong \mathbb{Z}[P] \oplus(\mathbb{Z} / 2 \mathbb{Z})([N]-[P])$. In particular $[N]-[P]$ is not algebraically trivial. The idea is that, for a closed point $O$ of $\mathbb{P}_{\mathbb{Q}}^{1}$ with residue field of degree $d$ over $\mathbb{Q}$, we have $\mathrm{Z}_{0}\left(\mathbb{P}_{\mathbb{Q}}^{1} \backslash O\right) /\left(\right.$ algebraic equivalence) $=\mathrm{CH}_{0}\left(\mathbb{P}_{\mathbb{Q}}^{1} \backslash O\right)=\mathbb{Z} / d \mathbb{Z}$ (this is proved using the localization exact sequence for Chow groups). Now, under the normalization morphism $\mathbb{P}_{\mathbb{Q}}^{1} \rightarrow X$, the pre-image of the node $N$ (which is a closed point with residue field $\mathbb{Q}$ ) is a closed point in $\mathbb{P}_{\mathbb{Q}}^{1}$ with residue field $\mathbb{Q}(i)$, and one can yet again use the localization exact sequence to conclude. Alternately, one could apply directly [Ful98, Exa. 1.8.1, Exa. 10.3.4] by considering the node sitting inside $X$.

Note in contrast that over $\mathbb{Q}(i)$ the same computation shows that the cycle $[N]-[P]$ is algebraically trivial, since the pre-image of the node in the normalization consists of two $\mathbb{Q}(i)$-points. As an aside, also note that $[N]$ and $[P]$ belong to the same component of the Chow scheme of $X / \mathbb{Q}$. Indeed, one can check that $X$ is semi-normal, so that by [Kol96, Exe. I.3.22] the Chow scheme Chow $_{0,1}(X)$ of 0 -dimensional subschemes of degree-1 in $X$ coincides with $X$. 
3.5. Abelian variety triviality. We now complete the proof of Theorem 1 by showing that curve triviality coincides with abelian variety triviality.

Proposition 3.14. Let $X$ be a scheme of finite type over a field $K$, and let $a \in \mathrm{CH}_{i}(X)$ be a projective curve trivial cycle class. Then a is abelian variety trivial. Thus, given Proposition 3.10, we have the following equivalences among the notions of triviality:

ab. var. triv. $\Longleftrightarrow$ proj. curve triv. $\Longrightarrow$ curve triv. $\Longleftrightarrow$ q.p. sch. triv. $\Longleftrightarrow$ sep. sch. triv. $\Longleftrightarrow$ alg. triv.

In particular, given Lemma 3.8, if $K$ is perfect, then all the notions of triviality above are equivalent.

Proof. By assumption there exist a smooth projective curve $C / K$, a cycle class $Z \in \mathrm{CH}_{i+1}\left(C \times_{K} X\right)$, and $K$-points $p_{1}, p_{0} \in C(K)$ such that $Z_{p_{1}}-Z_{p_{0}}=a \in \mathrm{CH}_{i}(X)$. Since $C$ has a $K$-point, $C$ is geometrically integral.

Let $g$ be the genus of $C$. Take $N>2 g-1$. Consider as in the proof of Lemma 3.9 the symmetrized cycle $S^{N} Z$ on $S^{N} C \times_{K} X$; its fiber over $r_{1}+\cdots+r_{N} \in S^{N} C$ is $\sum Z_{r_{i}}$. Let $q_{1}$ be the $K$-point of $S^{N} C$ corresponding to the divisor $N p_{1}$, and let $q_{0}$ be the $K$-point of $S^{N} C$ corresponding to $p_{0}+(N-1) p_{1}$. Then we have that $\left(S^{N} Z\right)_{q_{1}}-\left(S^{N} Z\right)_{q_{0}}=Z_{p_{1}}-Z_{p_{0}}=a$.

Since $N>2 g-1$, the Abel-Jacobi map

$$
A J_{N}: S^{N} C \rightarrow \operatorname{Pic}_{C / K}^{N}
$$

is a Zariski locally trivial fibration in projective spaces $\mathbb{P}_{K}^{N-g}$; i.e., there is a vector bundle $E$ of rank $N+1-g$ on $\operatorname{Pic}_{C / K}^{N}$ so that the Abel-Jacobi map coincides with the structure map $\mathbb{P} E \rightarrow \operatorname{Pic}_{C / K}^{N}$ (see e.g., [AK80, Thm. 8.5(v)] and [Kle05, Exe. 9.4.7]). Consequently, the map

$$
A J_{N} \times \mathrm{Id}: S^{N} C \times_{K} X \rightarrow \operatorname{Pic}_{C / K}^{N} \times_{K} X
$$

is the projectivization of the pull-back $E^{\prime}$ of $E$ to $\operatorname{Pic}_{C / K}^{N} \times_{K} X$. Denote by $\mathcal{O}(1)$ (resp. $\left.\mathcal{O}^{\prime}(1)\right)$ the relatively very ample line bundle on $S_{C / K}^{N}$ (resp. $\left.S_{C / K}^{N} \times_{K} X\right)$. Thanks to the projective bundle formula [Ful98, Thm. 3.3(b)] we can write

$$
S^{N} Z=\sum_{j=0}^{N+1-g} c_{1}\left(\mathcal{O}^{\prime}(1)\right)^{j} \cap\left(A J_{N} \times \mathrm{Id}\right)^{*} W_{j}
$$

for uniquely determined $W_{j} \in \mathrm{CH}_{i+1-(N-g)+j}\left(\mathrm{Pic}_{C / K}^{N} \times_{K} X\right)$. Now, we observe that the terms with $j$ positive in the above sum do not contribute to Gysin fibers over points of $S^{N} C$. More precisely, if $q: \operatorname{Spec} K \rightarrow S^{N} C$ is a $K$-point, we claim that

$$
q^{!} S^{N} Z=q^{!}\left(A J_{N} \times \mathrm{Id}\right)^{*} W_{0} .
$$

Indeed, since $\mathcal{O}^{\prime}(1)$ is obtained from $\mathcal{O}(1)$ by pull-back, then taking a cycle $D$ representing the cycle class $c_{1}(\mathcal{O}(1)) \cap\left[S_{C / K}^{N}\right]$, the associated intersections in the sum (3.6) can be taken to be supported on $\operatorname{Supp}(D) \times_{K} X$, and so the Gysin fibers must be zero. More precisely, with the notation in the cartesian diagram below:

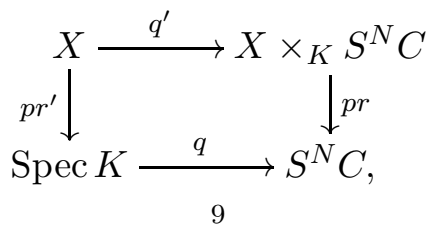


where $p r$ is the second projection, we have

$$
\begin{aligned}
q^{!}\left(c_{1}\left(\mathcal{O}^{\prime}(1)\right)^{j} \cap\left(A J_{N} \times \mathrm{Id}\right)^{*} W_{j}\right) & =c_{1}\left(q^{*} \mathcal{O}^{\prime}(1)\right)^{j} \cap q^{!}\left(A J_{N} \times \mathrm{Id}\right)^{*} W_{j} \quad \text { ([Ful98, Prop. 6.3]) } \\
& =c_{1}\left(q^{\prime *} p r^{*} \mathcal{O}(1)\right)^{j} \cap q^{!}\left(A J_{N} \times \mathrm{Id}\right)^{*} W_{j} \\
& =c_{1}\left(p r^{*} q^{*} \mathcal{O}(1)\right)^{j} \cap q^{!}\left(A J_{N} \times \mathrm{Id}\right)^{*} W_{j} \\
& =0 \cap q^{!}\left(A J_{N} \times \mathrm{Id}\right)^{*} W_{j}
\end{aligned}
$$

where the last equality holds since $q^{*} \mathcal{O}(1)=\mathcal{O}_{\text {Spec } K}$, and $c_{1}\left(\mathcal{O}_{X}\right)=0$.

Having established the claim, then setting $t=A J_{N} \circ q: \operatorname{Spec} K \rightarrow \operatorname{Pic}_{C / K}^{N}$ to be the composition, we have $q^{!} S^{N} Z=q^{!}\left(A J_{N} \times \mathrm{Id}\right)^{*} W_{0}=t^{!} W_{0}$ ([Ful98, Prop. 6.5(b)]). Therefore, setting $t_{j}=A J_{N} \circ q_{j}$ : Spec $K \rightarrow \operatorname{Pic}_{C / K}^{N}$, for $j=1,0$, we have

$$
Z_{p_{1}}-Z_{p_{0}}=\left(S^{N} Z\right)_{q_{1}}-\left(S^{N} Z\right)_{q_{0}}=\left(W_{0}\right)_{t_{1}}-\left(W_{0}\right)_{t_{0}} .
$$

Since $C$ has a $K$-point, $\operatorname{Pic}_{C / K}^{N}$ is an abelian variety, and we are done.

Remark 3.15. For comparison, in Appendix B we include Weil's argument in the case where $K=\bar{K}$ (see Lemma B.1). The key point is that by using the refined Gysin homomorphism, we are able to get by with less Brill-Noether theory; in particular, we do not need to assume the existence of Brill-Noether-general divisors of a given degree defined over the base field.

3.6. Algebraically trivial cycles and flat families. In the definition of algebraic equivalence for cycle classes (Definition 3.1), one uses refined Gysin homomorphisms. Those are only defined up to rational equivalence. This leads to the following definition of algebraic equivalence for cycles :

Definition 3.16 (Algebraically trivial cycle [Ful98, Def. 10.3]). Let $X$ be a scheme of finite type over a field $K$. A dimension- $i$ cycle $\alpha \in \mathrm{Z}_{i}(X)$ on $X$ is called algebraically trivial if its associated cycle class $a=[\alpha] \in \mathrm{CH}_{i}(X)$ is algebraically trivial.

We can also define a notion of algebraic equivalence for cycles using flat families. Given schemes of finite type $X$ and $T$ over $K$, integers $m_{j}$, and integral $\left(i+d_{T}\right)$-dimensional subschemes $Z_{j}$ of $T \times_{K} X$, a cycle $Z:=\sum_{j} m_{j} Z_{j} \in \mathrm{Z}_{i+d_{T}}\left(T \times_{K} X\right)$ is said to be flat over $T$ if each natural projection morphism $Z_{i} \rightarrow T$ is flat. If $T$ is smooth over $K$, and $t \in T(K)$, we define the flat fiber $Z_{t}$ of $Z$ over $t$ to be $\sum_{j} m_{j}\left(Z_{j}\right)_{t}$, where $\left(Z_{j}\right)_{t}$ is the scheme theoretic fiber over $t$. Note that in this situation, the flat fiber and the refined Gysin fiber agree. Indeed, in the case where $T$ is a smooth curve, this is asserted on the bottom of [Ful98, p.176]. More generally, one uses the formula for the Gysin fiber in terms of a Segre class given in [Ful98, p.176] (and [Ful98, Prop. 6.1(a)]), together with the flatness hypothesis and [Ful98, Exa. 10.1.2, Exa. A.5.5], so that one can use the fact that for a regular embedding, the Segre class can be given in terms of the Chern class of a normal bundle [Ful98, p.74].

Definition 3.17 (Flatly curve trivial cycle). Let $X$ be a scheme of finite type over a field $K$. A dimension- $i$ cycle $\alpha \in \mathrm{Z}_{i}(X)$ on $X$ is called flatly curve trivial if there exist a smooth integral curve $T$ over $K$, an effective cycle $Z \in \mathrm{Z}_{i+d_{T}}\left(T \times_{K} X\right)$ flat over $T$ (i.e., there are integral subschemes $V_{j} \subseteq T \times_{K} X$ flat over $T, 1 \leq j \leq r$, for some $r$, and $\left.Z=\sum_{i=1}^{r}\left[V_{i}\right]\right)$, and $K$-points $t_{1}, t_{0} \in T(K)$ such that $Z_{t_{1}}-Z_{t_{0}}=\alpha$ in $Z_{i}(X)$.

It is clear that if a cycle is flatly curve trivial, then it is algebraically trivial. The following lemma shows that the converse is true. It also shows that one obtains the same notion of triviality for cycles by allowing $T$ in Definition 3.17 to be an arbitrary smooth integral scheme of finite type over $K$.

Lemma 3.18. A cycle is algebraically trivial if and only if it is flatly curve trivial. 
Proof. The proof here extends [Fu198, Exa. 10.3.2] to the case where $K$ is not algebraically closed. Assume the cycle class $a$ is algebraically trivial. By Proposition 3.10, we may assume that $a$ is curve trivial. By definition of curve trivial, there exist a smooth quasi-projective integral curve $C^{\prime} / K$, a cycle class $Z^{\prime} \in \mathrm{CH}_{i+1}\left(C^{\prime} \times_{K} X\right)$, and $K$-points $p_{1}^{\prime}, p_{0}^{\prime} \in C^{\prime}(K)$ such that $Z_{p_{1}^{\prime}}^{\prime}-Z_{p_{0}^{\prime}}^{\prime}=a$ in $\mathrm{CH}_{i}(X)$.

Let $\widetilde{Z} \in \mathrm{Z}_{i+1}\left(C^{\prime} \times_{K} X\right)$ be a representative of $Z^{\prime}$. As in [Ful98, p.176], we may simply discard all components of $\widetilde{Z}$ that do not map dominantly onto $C^{\prime}$, and obtain a cycle $\widetilde{Z}$ all of whose components are flat over $C^{\prime}$, and with $[\widetilde{Z}]_{p_{1}^{\prime}}-[\widetilde{Z}]_{p_{0}^{\prime}}=[\alpha]$ in $\mathrm{CH}_{i}(X)$. The issue is that $\widetilde{Z}$ need not be nonnegative (there can be components of $\widetilde{Z}$ taken with negative coefficients). To rectify this, we argue as follows.

With $\widetilde{Z}$ as above, let $\widetilde{Z}=\widetilde{Z}^{+}-\widetilde{Z}^{-}$, with $\widetilde{Z}^{+}$and $\widetilde{Z}^{-}$effective, be the decomposition of $\widetilde{Z}$ into positive and negative parts. Set

$$
\begin{aligned}
S & =C^{\prime} \times_{K} C^{\prime}, \\
s_{1} & =\left(p_{1}^{\prime}, p_{0}^{\prime}\right) \in S(K), \\
s_{0} & =\left(p_{0}^{\prime}, p_{1}^{\prime}\right) \in S(K) \\
V^{+} & =\operatorname{pr}_{2}^{*} \widetilde{Z}^{+} \in \mathrm{CH}_{i+2}\left(S \times_{K} X\right) \\
V^{-} & =\operatorname{pr}_{1}^{*} \widetilde{Z}^{-} \in \mathrm{CH}_{i+2}\left(S \times_{K} X\right) \\
V & =V^{+}+V^{-} \in \mathrm{CH}_{i+2}\left(S \times_{K} X\right) .
\end{aligned}
$$

Since $C^{\prime}$ is quasi-projective, the same is true of $S$. Since $C^{\prime} / K$ is geometrically integral (see Remark 3.3), we have that $S$ is geometrically integral. Since $C^{\prime} / K$ is smooth, $C^{\prime} \times_{K} C^{\prime}$ is smooth. Finally, we have $V_{s_{1}}-V_{s_{0}}=Z_{p_{1}^{\prime}}-Z_{p_{0}^{\prime}} \in \mathrm{CH}_{i}(X)$.

To reduce to the case of a curve, we use Bertini's theorem (Theorem A.1). We obtain a smooth geometrically integral quasi-projective curve $g: C \hookrightarrow S$ over $K$, passing through the $K$-points $s_{1}, s_{0}$. We call these $K$-points $p_{1}, p_{0}$, respectively on $C$. The cycle class $Z=g^{!} V$ has the property that $Z_{p_{1}}-Z_{p_{0}}=[\alpha]$ in $\mathrm{CH}_{i}(X)$.

Before continuing, note that the support of $V$ is obtained from the support of $\widetilde{Z}$ by pull-back, so the support of $V$ is flat over $S$. Consequently, we may take scheme theoretic fibers of $V$ to obtain the Gysin fibers, and so we may assume that $Z$ is an effective cycle (not just a cycle class) with support flat over $C$. The only issue now is that we have $\left[Z_{p_{1}}\right]-\left[Z_{p_{0}}\right]=[\alpha]$ in $\mathrm{CH}_{i}(X)$, not as an equality of cycles.

Nevertheless, since the cycles $Z_{p_{1}}-Z_{p_{0}}$ and $\alpha$ are rationally equivalent, using [Ful98, Exa. 1.6.2], there is an effective cycle $W \in \mathrm{Z}_{i+1}\left(\mathbb{P}_{K}^{1} \times_{K} X\right)$ with all components flat over $\mathbb{P}_{K}^{1}$, a cycle $\beta \in \mathrm{Z}_{i}(X)$, and a pair of $K$-points $q_{0}, q_{1} \in \mathbb{P}_{K}^{1}(K)$ such that

$$
W_{q_{1}}=Z_{p_{1}}-Z_{p_{0}}+\beta, \quad W_{q_{0}}=\alpha+\beta .
$$

Now we take the product $C \times{ }_{K} \mathbb{P}_{K}^{1}$, pull-back our flat cycles $Z$ and $W$, and use Bertini. In the same way as before, we obtain a smooth geometrically integral curve, and a family of effective cycles with flat support over the curve, such that the fiber of the family at one point is

$$
\left[Z_{p_{0}}\right]+\left(\left[Z_{p_{1}}\right]-\left[Z_{p_{0}}\right]+\beta\right)
$$

and the fiber at the other point is

$$
\left[Z_{p_{1}}\right]+\alpha+\beta
$$

The difference of the fibers is then

$$
\left(\left[Z_{p_{1}}\right]+\alpha+\beta\right)-\left(\left[Z_{p_{0}}\right]+\left(\left[Z_{p_{1}}\right]-\left[Z_{p_{0}}\right]+\beta\right)\right)=\alpha,
$$

completing the proof. 
3.7. Rational triviality. For completeness, in this section we discuss the connection with rational triviality. The main point is that by requiring in Definition 3.4 that the parameter space $T$ be a rational curve, one simply obtains the trivial equivalence relation (see Remark 3.20). Moreover, while Definitions 3.1 and 3.16 use the definition of rational triviality to define algebraic triviality, we review here that one can also use the notion of flatly curve trivial cycles (which does not depend on the definition of rational triviality) to define rational triviality.

Definition 3.19. Let $X$ be a scheme of finite type over a field $K$. We say a cycle class $a \in \mathrm{CH}_{i}(X)$ is rational curve trivial if in the terminology of Definition 3.4, the parameter space $T$ can be taken to be isomorphic to (an open subset of) $\mathbb{P}_{K}^{1}$. A cycle $\alpha \in \mathrm{Z}_{i}(X)$ is rational curve trivial if its cycle class in $\mathrm{CH}_{i}(X)$ is.

Remark 3.20 ([Ful98, Prop. 1.6]). A cycle $\alpha \in \mathrm{Z}_{i}(X)$ is rational curve trivial if and only if it is rationally trivial. Equivalently, a cycle class $a \in \mathrm{CH}_{i}(X)$ is rational curve trivial if and only if $a=0$.

Of course, rational curve triviality still does not provide an alternative definition of rational triviality for cycles (since Definition 3.19 uses cycle classes). However, following [Ful98, Prop. 1.6] it is possible to do this using the notion of flatly curve trivial cycles.

Definition 3.21. Let $X$ be a scheme of finite type over a field $K$. We say a cycle $\alpha \in \mathrm{Z}_{i}(X)$ is flatly rational curve trivial if in the terminology of Definition 3.17, the parameter curve $T$ can be taken to be isomorphic to (an open subset of) $\mathbb{P}_{K}^{1}$.

Remark 3.22 ([Ful98, Prop. 1.6]). A cycle $\alpha \in \mathrm{Z}_{i}(X)$ on $X$ is flatly rationally curve trivial if and only if it is rationally trivial.

Finally we have the following observation:

Remark 3.23. Suppose in Lemma 3.9 that there is an open subset of a smooth rational curve passing through the support of the zero-cycle $\beta$. Then $Z_{\beta}$ is rational curve trivial; i.e., $Z_{\beta}=0 \in \mathrm{CH}_{i}(X)$. Indeed, in the proof of the lemma, one reduces to the case $T=C \subseteq \mathbb{P}_{K}^{1}$ is an open subset, in which case the symmetric product $S^{N} T$ is an open subset of $\mathbb{P}_{K}^{N}$. Consequently, the two $K$-points of $S^{N} T$ in the proof can be joined by an open subset of $\mathbb{P}_{K}^{1}$, and one concludes using Remark 3.20.

3.8. Algebraic triviality and base field. Let $X$ be a scheme of finite type over a field $K$. If $K / F$ is a finite extension of fields, then $X$ is also a scheme of finite type over $F$. Given a cycle class $a \in \mathrm{CH}_{i}(X)$, one may ask whether it is algebraically trivial viewing $X$ as a scheme over $K$, or as a scheme over $F$.

Proposition 3.24. Let $K / F$ be a finite extension of fields, let $X$ be a scheme of finite type over $K$, and let $a \in \mathrm{CH}_{i}(X)$ be a cycle class on $X$. If a is algebraically trivial on $X$, considered as a scheme of finite type over $F$, then it is algebraically trivial on $X$, considered as a scheme of finite type over $K$. Moreover, the converse holds, if one assumes that $K / F$ is a separable extension.

Proof. Assume $a$ is algebraically trivial on $X$, considered as a scheme of finite type over $F$. By Proposition 3.10, there are a smooth integral quasi-projective curve $C$ over $F, F$-points $t_{0}$ and $t_{1}$ in $C(F)$, and a cycle $Z$ in $C \times_{F} X$ such that $a=Z_{t_{1}}-Z_{t_{0}}$. Note that $C \times_{F} X=C_{K} \times_{K} X$, and note that since $C$ has an $F$-point, $C$ is geometrically integral, and so $C_{K}$ is (geometrically) integral. Pulling back the $F$-points $t_{0}$ and $t_{1}$ to $K$-points $p_{0}$ and $p_{1}$ of $C_{K}$, and viewing $Z$ as a cycle in $C_{K} \times_{K} X$, one has that $Z_{t_{i}}=Z_{p_{i}}$ so that $a=Z_{p_{1}}-Z_{p_{0}}$, i.e., that $a$ is algebraically trivial on $X$, considered as a scheme of finite type over $K$.

Conversely, assume $K / F$ is separable, and assume $a$ is algebraically trivial on $X$, considered as a scheme of finite type over $K$. By Proposition 3.10, there are a smooth integral quasi-projective 
curve $C$ over $K, K$-points $t_{0}$ and $t_{1}$ in $C(K)$, and a cycle $Z$ in $C \times_{K} X$ such that $a=Z_{t_{1}}-Z_{t_{0}}$. Since $K / F$ is a finite extension, $C$ is a curve over $F$ as well. Moreover, since $K / F$ is separable, $C$ is smooth over $F$. Viewing $C$ and $X$ as schemes over $F$ and $Z$ as a cycle in $C \times{ }_{F} X$ via the natural inclusion $C \times_{K} X \hookrightarrow C \times_{F} X$, one has that $a=Z_{\beta}$, where $\beta=\left[t_{1}\right]-\left[t_{0}\right] \in \mathrm{CH}_{0}(C)$. By Lemma 3.9, $a$ is algebraically trivial on $X$, considered as a scheme of finite type over $F$.

\section{Algebraic equivalence: Descending the parameter spaces}

For applications such as [ACMV17], our main result concerns a related notion of triviality for cycles after algebraic base change of field. More precisely, given a separable algebraic extension of fields $L / K$, a scheme $X$ of finite type over $K$, and an algebraically trivial cycle class $\alpha \in \mathrm{CH}_{i}\left(X_{L}\right)$, we show that triviality of $\alpha$ can be exhibited by a family of cycles defined over $K$ (although the fibers are taken over $L$-points).

4.1. $\rho$-, $\sigma$ - and $\tau$-algebraic triviality. We now introduce three additional notions of triviality.

Definition 4.1 ( $\rho$-, $\sigma$ - and $\tau$-algebraic triviality). Let $X$ be a scheme of finite type over a field $K$, and let $L$ be any field extension of $K$. A cycle class $a \in \mathrm{CH}_{i}\left(X_{L}\right)$ is said to be

- $\rho_{L / K^{-}}$algebraically trivial if there exist a smooth geometrically integral scheme $T$ of finite type over $K$, a cycle class $Z \in \mathrm{CH}_{i+d_{T}}\left(T \times_{K} X\right)$, and points $t_{0}, t_{1} \in T(L)$ such that $a=Z_{t_{1}}-Z_{t_{0}}$ in $\mathrm{CH}_{i}\left(X_{L}\right)$.

- $\sigma_{L / K}$-algebraically trivial if there exist a smooth geometrically integral scheme $T$ of finite type over $K$, a cycle class $Z \in \mathrm{CH}_{i+d_{T}}\left(T \times_{K} X\right)$, and points $t_{0}, t_{1} \in T(\bar{L})$ such that $a_{\bar{L}}=Z_{t_{1}}-Z_{t_{0}}$ in $\mathrm{CH}_{i}\left(X_{\bar{L}}\right)$.

- $\tau_{L / K^{-}}$algebraically trivial if $N a$ is $\rho_{L / K^{-}}$-algebraically trivial for some non-zero integer $N$; i.e., there exist a smooth geometrically integral scheme $T$ of finite type over $K$, a cycle class $Z \in \mathrm{CH}_{i+d_{T}}\left(T \times_{K} X\right)$, and points $t_{0}, t_{1} \in T(L)$ such that $N a=Z_{t_{1}}-Z_{t_{0}}$ in $\mathrm{CH}_{i}\left(X_{L}\right)$.

Remark 4.2. In the above definition, the morphisms $t_{i}$ : Spec $L \rightarrow T$ need not be closed embeddings, or even morphisms of finite type over $K$, and so the refined Gysin fiber $Z_{t_{i}}$ of [Ful98, §6.2] is not immediately well-defined. In the context of the conventions in [Ful98], what we mean is the following. For a finite extension $L / K$, the morphism $t_{i}$ : Spec $L \rightarrow T$ is an lci morphism, in the strong sense of [Ful98, B.7.6]; then we can define the refined Gysin fiber as in [Ful98, §6.6]. For an arbitrary extension $L / K$, the field $L$ can be written as a direct limit $L=\underset{\lim }{\longrightarrow}$ of $K$-algebras of finite type. For each $R$, using flat pull-back one obtains a cycle class $Z_{T_{R}} \in \mathrm{CH}^{i}\left(\left(T \times_{K} X\right)_{R}\right)$ and one obtains a cycle class $Z_{T_{L}} \in \mathrm{CH}^{i}\left(\left(T \times_{K} X\right)_{L}\right)$ using the identification $\mathrm{CH}^{i}\left(\left(T \times_{K} X\right)_{L}\right)=$ $\left.\lim _{\mathrm{CH}^{i}}\left(\left(T \times_{K} X\right)_{R}\right)\right)$. Then one can take $Z_{t_{i}}$ to be the refined Gysin fiber of $Z_{L}$ over the closed

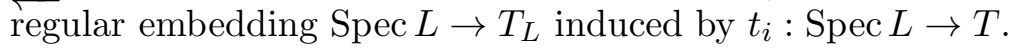

Remark 4.3. It is easy to see that a $\rho_{L / K}$-algebraically trivial cycle class is algebraically trivial; moreover, if $L=K$, then algebraic triviality is by definition the same as $\rho_{K / K^{-a l g e b r a i c}}$ triviality. It is clear that a $\rho_{L / K}$-algebraically trivial cycle class is $\sigma_{L / K}$-algebraically trivial. Over an algebraically closed field $K=\bar{K}$, algebraic triviality is by definition the same as $\sigma_{K / K}$-algebraic triviality. Our main result, Theorem 4.11, compares all these notions.

Remark 4.4. Over an arbitrary field $K$, an algebraically trivial cycle class $a \in \mathrm{CH}_{i}(X)$ is by definition both $\sigma_{K / K^{-}}$and $\tau_{K / K^{-}}$algebraically trivial. The converse fails: the rational nodal curve over $\mathbb{Q}$ of Example 3.13 with the 0 -cycle $[N]-[P]$ provides a counter-example. Indeed, $2([N]-[P])$ is algebraically trivial, so that $[N]-[P]$ is $\tau_{K / K^{-}}$algebraically trivial, and $[N]-[P]$ becomes rationally trivial after base-changing to an algebraic closure of $\mathbb{Q}$, so that $[N]-[P]$ is also $\sigma_{K} / K^{\text {-algebraically }}$ trivial. However, $[N]-[P]$ is not algebraically trivial. 
Remark 4.5. In [Kle68], one finds the notion of $\tau$-algebraically trivial cycles (see also [Ful98, p.374]); in our notation, this is $\tau_{K / K}$-algebraic triviality.

Definition 4.6. Similarly to Definition 3.4, one may define refined notions of $\rho$-, $\sigma$ - and $\tau$-algebraic triviality, e.g., $\sigma_{L / K^{-}}$abelian variety trivial, $\tau_{L / K^{-}}$curve trivial, etc.

In the next subsections we will establish further relationships among the notions of triviality.

4.2. First properties of $\rho$-, $\sigma$ - and $\tau$-triviality. We start by extending the classical results in $\S 3$ to the new notions of triviality.

Proposition 4.7. For any of the notions of triviality in Definition 4.1 and Definition 4.6 above, the trivial cycles classes form a subgroup of the group of all cycle classes. In the category of schemes of finite type over $K$, this subgroup is preserved by the basic operations:

(1) proper push-forward;

(2) flat pull-back;

(3) refined Gysin homomorphisms;

(4) Chern class operations.

Proof. The proof is the same as [Fu198, Prop. 10.3]. In the case of curves, we in addition use Bertini's theorem to cut the product of curves back down to a curve. That the four operations (1)-(4) are preserved follows from the corresponding parts (a)-(d) of [Ful98, Prop. 10.1].

Remark 4.8. By virtue of the previous proposition, given a commutative ring $R$, we extend the notions of triviality in Definitions 4.1 and 4.6 to Chow groups with $R$-coefficients by extending $R$-linearly.

Remark 4.9. A cycle $a \in \mathrm{CH}_{i}\left(X_{L}\right)$ is $\tau_{L / K^{-}}$-algebraically trivial if and only if $a$, viewed as an element of $\mathrm{CH}_{i}\left(X_{L}\right)_{\mathbb{Q}}$, is $\rho_{L / K}$-algebraically trivial.

We also have the following analogue of Theorem 1.

Proposition 4.10. Let $X$ be a scheme of finite type over a field $K$, let $L / K$ be an algebraic extension of fields, and let $R$ be a commutative ring. (If $K$ is imperfect of characteristic $p$, assume that $p$ is invertible in $R$.) If $a \in \mathrm{CH}_{i}\left(X_{L}\right)_{R}$ is a $\rho_{L / K^{-}}\left(\right.$resp. $\sigma_{L / K^{-}}$, resp. $\left.\tau_{L / K^{-}}\right)$algebraically trivial cycle class, then a is $\rho_{L / K^{-}}$(resp. $\sigma_{L / K^{-}}$, resp. $\left.\tau_{L / K^{-}}\right)$abelian variety trivial. Moreover, we have the following implications among the notions of triviality (with $R$-coefficients):

$$
\begin{aligned}
\rho_{L / K^{-}} \text {ab. var. } \Longleftrightarrow \rho_{L / K^{-}} \text {curve } \Longleftrightarrow \rho_{L / K^{-}} \text {q.p. sch. } \Longleftrightarrow \rho_{L / K^{-}} \text {sep. sch. } \Longleftrightarrow \rho_{L / K^{-}} \text {alg. } \\
\sigma_{L / K^{-}} \text {ab.var. } \Longleftrightarrow \sigma_{L / K^{-}} \text {curve } \Longleftrightarrow \sigma_{L / K^{-}} \text {.p.p.sch. } \Longleftrightarrow \sigma_{L / K^{-}} \text {sep. sch. } \Longleftrightarrow \sigma_{L / K^{-}} \text {alg. } . \\
\tau_{L / K^{-}} \text {ab.var. } \Longleftrightarrow \tau_{L / K^{-}} \text {curve } \Longleftrightarrow \tau_{L / K^{-}} \text {q.p. sch. } \Longleftrightarrow \tau_{L / K^{-}} \text {sep. sch. } \Longleftrightarrow \tau_{L / K^{-}} \text {alg. }
\end{aligned}
$$

Proof. As in Remark 3.7, using Bertini's Theorem, we see that the implications from left to right hold. The proof of Proposition 3.10 can be easily adapted to show that $\rho_{L / K^{-}}\left(\right.$resp. $\sigma_{L / K^{-}}$, resp. $\left.\tau_{L / K^{-}}\right)$triviality implies $\rho_{L / K^{-}}\left(\right.$resp. $\sigma_{L / K^{-}}$, resp. $\left.\tau_{L / K^{-}}\right)$curve triviality. The proof of Proposition 3.14 can also be easily adapted to show (using Lemma 3.8 if $K$ is imperfect) that $\rho_{L / K^{-}}$ (resp. $\sigma_{L / K^{-}}$, resp. $\left.\tau_{L / K^{-}}\right)$curve triviality implies $\rho_{L / K^{-}}\left(\right.$resp. $\sigma_{L / K^{-}}$, resp. $\left.\tau_{L / K^{-}}\right)$abelian variety triviality with $R$-coefficients.

4.3. Main theorem. Our main result is to compare algebraic triviality with $\rho$-, $\sigma$ - and $\tau$-algebraic triviality: 
Theorem 4.11. Let $X$ be a scheme of finite type over a field $K$, let $L$ be an algebraic extension of $K$, and let $R$ be a commutative ring. Consider a cycle class a $\in \mathrm{CH}_{i}\left(X_{L}\right)_{R}$, and consider the following statements :

(i) The cycle a is algebraically trivial;

(ii) The cycle a is $\rho_{L / K}$-algebraically trivial;

(iii) The cycle a is $\sigma_{L / K}$-algebraically trivial;

(iv) The cycle a is $\tau_{L / K}$-algebraically trivial.

Then (ii) $\Longrightarrow$ (i) and (ii) $\Longrightarrow$ (iii) $\Longrightarrow$ (iv). If in addition one assumes that either $L / K$ is separable or char $(K)$ is invertible in $R$, then (i) $\Longrightarrow$ (ii).

Note from Remark 4.3 that the only implications left to show in Theorem 4.11 are the implication (iii) $\Longrightarrow$ (iv), and the implication (i) $\Longrightarrow$ (ii). These are proved respectively in Lemma 4.15, and Lemma 4.14. Note that Theorem 2 is the implication (i) $\Longrightarrow$ (ii) of Theorem 4.11 in the case where $L / K$ is separable. Note also that complex Enriques surfaces provide counter-examples to the implication (iv) $\Longrightarrow$ (iii) (see Example 4.12 below), and that the rational nodal curve over $\mathbb{Q}$ of Example 3.13 provides a counter-example to the implication (iii) $\Longrightarrow$ (ii) (see Remark 4.4).

Example 4.12. In general, $\tau_{L / K}$-triviality does not imply $\sigma_{L / K}$-triviality. Consider for instance a non-zero torsion element in the Néron-Severi group of a smooth complex projective variety (e.g.,

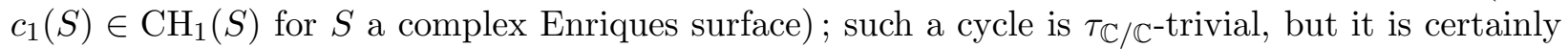
not $\sigma_{\mathbb{C} / \mathbb{C}^{-t r i v i a l . ~}}$

Corollary 4.13. Let $X$ be a scheme of finite type over a field $K$, let $L$ be a separable algebraic extension of $K$, and let $R$ be a commutative ring. For cycle classes in $\mathrm{CH}_{i}\left(X_{L}\right)_{R}$ :

$$
\text { alg. triv. } \Longleftrightarrow \rho_{L / K^{-}} \text {alg. triv. } \Longrightarrow \sigma_{L / K^{-}} \text {alg. triv. } \Longrightarrow \tau_{L / K^{-}} \text {alg.triv. }
$$

Moreover, if $K=\bar{K}$ then the middle implication is an equivalence, and if $\mathbb{Q} \subseteq R$ then all implications are equivalences.

\subsubsection{Proof of (i) $\Longrightarrow$ (ii) in Theorem 4.11.}

Lemma 4.14. Let $X$ be a scheme of finite type over a field $K$, let $L / K$ be an algebraic field extension, let $R$ be a commutative ring, and let $a \in \mathrm{CH}_{i}\left(X_{L}\right)_{R}$ be an algebraically trivial cycle class with $R$-coefficients. Assume either that $L / K$ is separable, or that $\operatorname{char}(K)$ is invertible in $R$.

Then there are a smooth geometrically integral curve $C / K$, admitting a $K$-point, a cycle class $Z \in \mathrm{CH}_{i+1}\left(C \times_{K} X\right)$, and L-points $p_{1}$, $p_{0}$ on $C$ such that $Z_{p_{1}}-Z_{p_{0}}=a_{L}$ in $\mathrm{CH}_{i}\left(X_{L}\right)_{R}$. In other words, a is $\rho_{L / K}$-curve trivial.

Proof. It is easy to see that there is a finite field extension $F / K$ and an algebraically trivial cycle $a_{F} \in \mathrm{CH}_{i}\left(X_{F}\right)_{R}$ such that $a=\left(a_{F}\right)_{L}$. Thus we may assume that $L / K$ is finite. In the case where $K$ has positive characteristic, say $p$, denote $L^{s e p}$ the largest separable extension of $K$ contained in $L$ and let $p^{r}$ be the degree of the purely inseparable extension $L / L^{s e p}$. Then, for the natural projection proper morphism $f: X_{L} \rightarrow X_{L^{s e p}}$, we have $p^{r} a=f^{*} f_{*} a$. Therefore, if $p$ is invertible in $R$, it is enough to show that $f_{*} a$ is $\sigma_{L^{\text {sep }} / K^{-}}$-curve trivial. Thus it suffices to prove the lemma for the special case where $R=\mathbb{Z}$ and $L / K$ is a finite separable extension.

The proof is similar in spirit to that of Proposition 3.24 (together with Lemma 3.9), but slightly different in the sense that the Gysin fibers are taken over $L$-points rather than $K$-points. We proceed in two steps.

(1) By Proposition 3.10, there exist a smooth integral quasi-projective curve $D / L$, a cycle class $Y \in \mathrm{CH}_{i+1}\left(D \times{ }_{L} X_{L}\right)$, and $L$-points $q_{1}, q_{0}$ on $D$ such that $Y_{q_{1}}-Y_{q_{0}}=a_{L}$. Now since $L / K$ is 
finite and separable, $D$ is a smooth quasi-projective curve over $K$ as well. Now we make the trivial observation that

$$
D \times_{L} X_{L}=D \times_{L}\left(L \times_{K} X\right)=D \times_{K} X .
$$

Therefore, $Y \in \mathrm{CH}_{i+1}\left(D \times_{K} X\right)$, and, taking fibers over $L$-points, we have $Y_{q_{1}}-Y_{q_{0}}=a_{L}$.

(2) Viewed as a smooth curve over $K, D$ is integral but, if $K \subsetneq L, D$ is not geometrically irreducible, and $D(K)$ is empty. Nonetheless, as in the proof of Lemma 3.9, we can identify a geometrically irreducible curve (over $K$ ) inside a suitable symmetric power of $D$. Let $e:=[L: K]$; then $D_{L}=D \times_{K} L$ has $e$ irreducible components $\bar{D}^{(1)}, \cdots, \bar{D}^{(e)}$, each of which is geometrically irreducible. Inside the $e$-fold symmetric product $S^{e}(D)$ of $D$ (over $K$ ), we have the geometrically irreducible subvariety $S^{\Delta_{1}}(D)$ (Lemma 2.1); and each $q_{i} \in D(L)$ gives rise to a point $\left\{x_{i}^{1}, \cdots, x_{i}^{e}\right\} \in$ $S^{\Delta_{1}}(D)(K)$, where $x_{i}^{j} \in \bar{D}^{(j)}$.

Now let $S^{e} Y$ be the symmetrized induced cycle on $S^{e}(D) \times_{K} X$ and let $Z$ be the restriction of $S^{e} Y$ to $S^{\Delta_{1}}(D) \times X$. Let $p_{1}=\left\{x_{1}^{1}, x_{1}^{2}, \cdots, x_{1}^{e}\right\} \in S^{\Delta_{1}}(D)(K) \subset S^{\Delta_{1}}(D)(L)$ and let $q_{1}=$ $\left\{x_{0}^{1}, x_{0}^{2}, x_{0}^{3}, \cdots, x_{0}^{e}\right\} \in S^{\Delta_{1}}(D)(L)$. Then $Z_{p_{1}}-Z_{p_{0}}=Y_{q_{1}}-Y_{q_{0}}=a_{L} \in \mathrm{CH}\left(X_{L}\right)$. To conclude the proof we use Bertini's theorem (Theorem A.1) to obtain a geometrically integral smooth curve $C$ in $S^{\Delta_{1}}(D)$ passing through $p_{1}$ and $p_{0}$.

\subsubsection{Proof of (iii) $\Longrightarrow$ (iv) in Theorem 4.11. In view of Proposition 4.10, it suffices to prove:}

Lemma 4.15. Let $X$ be a scheme of finite type over $K$, let $L / K$ be an algebraic extension, and let $a \in \mathrm{CH}_{i}\left(X_{L}\right)$ be a cycle class. Suppose there exist a finite extension $L^{\prime} / L$, a smooth geometrically integral curve $C / K$, a cycle class $Z \in \mathrm{CH}_{i+1}\left(C \times_{K} X\right)$ and $L^{\prime}$-points $p_{1}, p_{0} \in C\left(L^{\prime}\right)$ such that $Z_{p_{1}}-Z_{p_{0}}=a_{L^{\prime}}$ in $\mathrm{CH}_{i}\left(X_{L^{\prime}}\right)$.

Then there exist a non-zero integer $N$, a smooth geometrically integral projective curve $C^{\prime} / K$, a cycle class $Z^{\prime} \in \mathrm{CH}_{i+1}\left(C^{\prime} \times_{K} X\right)$ and L-points $q_{1}, q_{0} \in C(L)$ such that $Z_{q_{1}}^{\prime}-Z_{q_{0}}^{\prime}=N a$ in $\mathrm{CH}_{i}\left(X_{L}\right)$.

Proof. Denote pr: $X_{L^{\prime}} \rightarrow X_{L}$ the natural projection, and denote $\left[p_{i}\right]$ the push-forward of the fundamental class of $p_{i}, i=0,1$, to $C$. By definition of the fiber of a cycle above a zero-cycle (see Section 3.4), we have that $\operatorname{pr}_{*}\left(Z_{p_{1}}-Z_{p_{0}}\right)=Z_{\left[p_{1}\right]}-Z_{\left[p_{0}\right]}$. On the other hand, $\operatorname{pr}_{*}\left(a_{L^{\prime}}\right)=\left[L^{\prime}: L\right] a$ by the projection formula. Applying a variant of Lemma 3.9 with $L$-points rather than $K$-points yields that $\left[L^{\prime}: L\right] a$ is $\rho_{L / K^{-}}$-algebraically trivial, and parameterized by a smooth quasi-projective geometrically integral scheme $T$. By Bertini's theorem (Theorem A.1), we may take $T$ to be a geometrically integral curve. In fact, if $K$ is perfect, we may take $T$ be be a geometrically integral projective curve. If $K$ is imperfect of characteristic $p$, then after replacing $\left[L^{\prime}: L\right] a$ by $p^{n}\left[L^{\prime}: L\right] a$ for some $n$, we may use Lemma 3.8 to arrive at the same conclusion.

This completes the proof of the main Theorem 4.11 .

4.4. Singular parameter spaces. In this last section, we discuss a notion of algebraic triviality where one allows for singular parameter spaces. In Section 3.6 we discussed an equivalent definition of algebraic triviality based on flat families of cycles and scheme theoretic fibers. There we required that the parameter space for the cycles be smooth, which was necessary in light of Example 3.13 (see Remark 4.19, below). In that example, however, the cycle causing the conflict was evidently both $\sigma_{K / K^{-}}$and $\tau_{K / K^{-}}$algebraically trivial. Here we show that when the ground field $K$ is perfect, this is always the case (Proposition 4.18).

Definition 4.16. Let $X$ be a scheme of finite type over a field $K$. A cycle $\alpha \in \mathrm{Z}_{i}(X)$ is said to be flatly trivial if there exists a (not necessarily smooth) integral scheme $T$ of finite type over $K$, an effective cycle $Z \in \mathrm{Z}_{i+d_{T}}\left(T \times_{K} X\right)$ flat over $T$ (i.e., there are integral subschemes $V_{j} \subseteq T \times_{K} X$ flat over $T, 1 \leq j \leq r$, for some $r$, and $\left.Z=\sum_{i=1}^{r}\left[V_{i}\right]\right)$, and $K$-points $t_{1}, t_{0} \in T(K)$ such that $Z_{t_{1}}-Z_{t_{0}}=\alpha$ in $Z_{i}(X)$. A cycle class is flatly trivial if it is the class of a cycle that is flatly trivial. 
Remark 4.17. An argument similar to that in Lemma 3.18 shows that if a cycle class $a \in \mathrm{CH}_{i}(X)$ is flatly trivial, then for any cycle $\alpha$ such that $a=[\alpha]$, we have that $\alpha$ is flatly trivial.

Proposition 4.18. Let $X$ be a scheme of finite type over a field $K$, and let $a \in \mathrm{CH}_{i}(X)$ be a cycle class.

(1) If a is algebraically trivial, then a is flatly trivial.

(2) Assume $K$ is perfect. If a is flatly trivial, then a is $\sigma_{K / K^{-a l g e b r a i c a l l y ~} \text { trivial (and therefore }}$ also $\tau_{K / K^{-}}$algebraically trivial).

Proof. Item (1) follows immediately from Lemma 3.18. As for item (2), consider a cycle $\alpha$ representing $a$ together with $T, Z, t_{1}$ and $t_{0}$ as in Definition 4.16. Since we are assuming that $K$ is perfect, there is by [dJ96] an alteration $\widetilde{T} \rightarrow T$ with $\widetilde{T} \rightarrow K$ smooth. Let $\tilde{t}_{1}$ and $\tilde{t}_{0}$ be any two $\bar{K}$-points in $\widetilde{T}$ lifting the points $t_{1}, t_{0} \in T(K)$, and let $\widetilde{Z}$ be the flat effective cycle on $\widetilde{T} \times_{K} X$ that is the pre-image of the cycle $Z$. Then it is clear that $a_{\bar{K}}=\widetilde{Z}_{\tilde{t}_{1}}-\widetilde{Z}_{\tilde{t}_{0}}$ in $\mathrm{CH}_{i}\left(X_{\bar{K}}\right)$, i.e., $a$ is $\sigma_{K / K^{-} \text {trivial. }}$

Remark 4.19. The converse of Proposition 4.18 (1) does not hold in general, as is shown in Example 3.13. Indeed, the cycle class $[N]-[P]$ is flatly trivial, via the diagonal in the product of the curve with itself. However, the cycle class is not algebraically trivial, as shown in the example. Note that $[N]-[P]$ is clearly both $\sigma_{K / K^{-}}$and $\tau_{K / K^{-}}$algebraically trivial, agreeing with the assertion of Proposition 4.18.

\section{Appendix A. A review of Bertini theorems}

We start by recalling a consequence of the Bertini theorems and Chow's theorem. For algebraically closed fields, this is [Mum70, Lem. p.56]. It has long been understood how to pass to arbitrary infinite fields; by [Poo04, CP16], the results are now known to hold over an arbitrary field. In particular, even over a finite field, one knows that every geometrically irreducible separated scheme of finite type contains a geometrically integral curve. In this paper, we use the case where the ambient scheme is smooth, which follows from [Poo04]; for the sake of completeness, we include a more general statement that uses [CP16].

Theorem A.1 (Bertini-Charles-Poonen). Let $X$ be an irreducible separated scheme of $\operatorname{dim} \geq 1$ of finite type over a field $K$, and let $\bar{x}_{1}, \ldots, \bar{x}_{n} \in X(\bar{K})$ be $\bar{K}$-points of $X$. There is an integral curve $C \subseteq X$ defined over $K$ containing the image of $\bar{x}_{1}, \ldots, \bar{x}_{n}$. Moreover :

(1) There exists such a $C$ so that there is a bijection between the geometric components of $C$ and the geometric components of $X$, with the property that every component of $X_{\bar{K}}$ contains exactly one component of $C_{\bar{K}}$.

(2) If all of the points $\bar{x}_{1}, \ldots, \bar{x}_{n}$, viewed as $\bar{K}$-points of $X_{\bar{K}}$, lie on the same component of $X_{\bar{K}}$, there exists such a $C$ such that all of the points $\bar{x}_{1}, \ldots, \bar{x}_{n}$, viewed as $\bar{K}$-points of $C_{\bar{K}}$, lie on the same component of $C_{\bar{K}}$.

(3) If $X$ is quasi-projective and $\operatorname{dim} X \geq 2$, there exists such a $C$ that is a general linear section of $X$ for some embedding of $X$ in a projective space.

(4) If $X$ is quasi-projective and smooth over $K$, there exists such a $C$ that is smooth over $K$, and we may further specify the tangent directions of $C$ inside $X$ at the points $\bar{x}_{1}, \ldots, \bar{x}_{n}$.

Proof. The case where $K=\bar{K}$ is [Mum70, Lem. p.56]. The case where $X$ is assumed to be geometrically integral is [Poo04, Thm. 1.1] and [CP16, Cor. 1.9]. The general case is essentially the same, but since the statements above require combining a few results, we include the brief proof.

We may always work with the reduced scheme structure, to assume that $X$ is integral. From Nagata's compactification theorem (e.g., [Con07]) $X$ admits an open immersion into a proper scheme $X^{\prime}$ over $K$. We may assume that $X^{\prime}$ is integral (for instance, by taking the reduced scheme 
structure). The geometric components of $X^{\prime}$ are in bijection with the geometric components of $X$. By Chow's theorem we may dominate $X^{\prime}$ with a projective scheme $X^{\prime \prime}$, birational to $X^{\prime}$. We can assume that $X^{\prime \prime}$ is integral (for instance, by taking reductions and components). Again, since $X^{\prime \prime}$ is birational to $X^{\prime}$, the geometric components of $X^{\prime}$ and $X^{\prime \prime}$ are in bijection.

Now lift the $\bar{K}$-points $\bar{x}_{1}, \ldots, \bar{x}_{n}$ of $X$ to $\bar{K}$-points $\bar{x}_{1}^{\prime \prime}, \ldots, \bar{x}_{n}^{\prime \prime}$ of $X^{\prime \prime}$, with $\bar{x}_{i}^{\prime \prime}(i=1, \ldots, n)$ lying on the component of $X_{\bar{K}}^{\prime \prime}$ corresponding to the component of $X_{\bar{K}}$ on which $\bar{x}_{i}$ lies. Using the Bertini irreducibility theorems ([Jou83, Thm. 6.3(4)], [CP16, Thm. 1.2 and Rem. 1.2(d)]) we may find $C^{\prime \prime} \subseteq X^{\prime \prime}$ an integral curve that is a general linear section of $X^{\prime \prime}$ for some projective embedding of $X^{\prime \prime}$, with $C^{\prime \prime}$ passing through the images of the points $\bar{x}_{1}^{\prime \prime}, \ldots, \bar{x}_{n}^{\prime \prime}$. Moreover, we may do this in such a way that there is a bijection between the geometric components of $C^{\prime \prime}$ and the geometric components of $X^{\prime \prime}$, with the property that every component of $X_{K}^{\prime \prime}$ contains exactly one component of $C_{\bar{K}}^{\prime \prime}$. Taking $C$ to be the restriction to $X$ of the image of $C^{\prime \prime}$ in $X^{\prime}$ provides the desired integral curve, and also establishes (1) and (2).

Finally, if $X$ is quasi-projective, then $X^{\prime \prime}$ above can be taken to be a projective completion of $X$. Then (3) follows from the argument above, and (4) follows from the Bertini theorems ([Poo04, Thm. 1.1, Thm. 1.2] for the case of finite fields).

If $X$ is not assumed to be separated, the theorem can fail :

Example A.2. Given a scheme $X$ of finite type over a field $K$, and $\bar{K}$-points $\bar{x}_{1}, \bar{x}_{2} \in X(\bar{K})$, if $X$ is not separated over $K$ there may be no integral curve $C \subseteq X$ containing the image $\bar{x}_{1}$ and $\bar{x}_{2}$. Indeed, take $X$ to be the non-separated scheme obtained from two copies of $\mathbb{A}_{K}^{1}$ by identifying all corresponding points but the origin, and take $\bar{x}_{1}$ and $\bar{x}_{2}$ to be the two copies of the origin.

However, even if $X$ is not assumed to be separated, the theorem does still imply the existence of chains of curves interpolating between points:

Corollary A.3. Let $X$ be an irreducible scheme of finite type over a field $K$ of $\operatorname{dim} \geq 1$, and let $\bar{x}_{1}, \bar{x}_{0} \in X(\bar{K})$ be $\bar{K}$-points of $X$. There is a chain of integral curves in $X$ containing the images of $\bar{x}_{1}, \bar{x}_{0}$. More precisely, there are integral curves $C_{1}, \ldots, C_{r} \subseteq X$ defined over $K$ for some $r$, so that for each $i=1, \ldots, r$ there are $\bar{K}$-points $\bar{x}_{i_{1}}, \bar{x}_{i_{0}} \in C_{i}(\bar{K})$, with $\bar{x}_{1}=\bar{x}_{1_{1}}, \bar{x}_{0}=\bar{x}_{r_{0}}$ and $\bar{x}_{i_{0}}=\bar{x}_{(i+1)_{1}}$ for $i=1, \ldots, r-1$. Moreover:

(1) There exists such a chain $C_{1}, \ldots, C_{r}$ so that for each $j$, the geometric components of $C_{j}$ are in bijection with the geometric components of $X$, and every component of $X_{\bar{K}}$ contains exactly one component of $\left(C_{j}\right)_{\bar{K}}$ for $j=1, \ldots, r$.

(2) If the points $\bar{x}_{1}, \bar{x}_{0}$, viewed as $\bar{K}$-points of $X_{\bar{K}}$, lie on the same component of $X_{\bar{K}}$, there exists such a chain $C_{1}, \ldots, C_{r}$ such that the points $\bar{x}_{1}, \bar{x}_{0}$, viewed as $\bar{K}$-points of $\left(C_{1}\right)_{\bar{K}}$ and $\left(C_{r}\right)_{\bar{K}}$, lie on the same connected component of the union $C_{\bar{K}}=\bigcup_{i=1}^{r}\left(C_{i}\right)_{\bar{K}}$.

(3) If $X$ is smooth over $K$ and $\operatorname{dim} X \geq 2$, there exists such a chain $C_{1}, \ldots, C_{r}$ such that the union $C=\bigcup_{i=1}^{r} C_{i}$ is a nodal curve, and such that the image of each point $\bar{x}_{2}, \ldots, \bar{x}_{r-1}$ has finite separable residue field over $K$.

Proof. Since $X$ is of finite type over $K$, we may cover $X$ with finitely many quasi-projective open subsets $U_{1}, \ldots, U_{r}$. Label these so that $\bar{x}_{1} \in U_{1}$ and $\bar{x}_{0} \in U_{r}$. Set $\bar{x}_{1_{1}}=\bar{x}_{1}$. We label new points inductively. If $r=1$, then set $\bar{x}_{1_{0}}=\bar{x}_{0}$. Otherwise, if $r>1$, to avoid indexing trivialities, let $\bar{x}_{1_{0}} \in U_{1} \cap U_{2}$ be a $\bar{K}$-point of $U_{1}$ that is different from $\bar{x}_{1_{1}}$. Set $x_{2_{1}}=x_{1_{0}}$. Continue inductively defining points in this way until we have $\bar{x}_{r_{0}}=\bar{x}_{0} \in U_{r}$. Now we may use Theorem A.1 to find curves $C_{i} \subseteq U_{i}$ passing through the given points, and with the asserted properties. (For the final claim of (3), use the fact that closed points with residue field finite and separable over the base field are Zariski dense in any geometrically reduced scheme which is locally of finite type.) 


\section{APPEndix B. WeIL's ARgument}

For comparison with Proposition 3.14, we include Weil's argument that over an algebraically closed field, curve triviality and abelian variety triviality agree. In fact, with Definitions 4.1 and 4.6 in mind, Weil's argument shows that $\rho_{L / K}$-curve triviality implies $\sigma_{L / K^{-}}$-abelian variety triviality for perfect fields $K$. The key point is that the Brill-Noether theory utilized in his argument requires the existence of divisors over the base field, which cannot in general be assumed to exist.

Lemma B.1 ([Wei54, p.108], [Lan59, p.60]). Let $X$ be a scheme of finite type over $K$, let $L / K$ be an algebraic extension, and let $a \in \mathrm{CH}_{i}\left(X_{L}\right)$ be a cycle class. Suppose there exist a smooth geometrically integral projective curve $C / K$ of genus $g$, a cycle class $Z^{\prime} \in \mathrm{CH}_{i+1}\left(C \times_{K} X\right)$ and $L$-points $p_{1}, p_{0} \in C(L)$ such that $Z_{p_{1}}^{\prime}-Z_{p_{0}}^{\prime}=a$ in $\mathrm{CH}_{i}\left(X_{L}\right)$.

Then there exist a cycle class $Z \in \mathrm{CH}_{i+g}\left(\mathrm{Pic}_{C / K}^{g} \times_{K} X\right)$, a finite separable field extension $L^{\prime} / L$, and $L^{\prime}$-points $t_{1}, t_{0} \in \operatorname{Pic}_{C / K}^{g}\left(L^{\prime}\right)$ such that $Z_{t_{1}}-Z_{t_{0}}=a_{L^{\prime}}$ in $\mathrm{CH}_{i}\left(X_{L^{\prime}}\right)$.

Remark B.2. If $C$ admits a $K$-point (or more generally a line bundle of degree $g$ over $K$ ), then $\mathrm{Pic}_{C / K}^{g}$ is isomorphic to an abelian variety, namely $\mathrm{Pic}_{C / K}^{0}$.

Proof. If $g=0$, then $a=0 \in \mathrm{CH}_{i}\left(X_{L}\right)$, and there is nothing to do, as we can for instance take $Z$ to be the zero cycle class. Otherwise, consider the $g$-fold symmetric product $S^{g} C$ together with the induced cycle $S^{g} Z^{\prime}$ on $S^{g} C \times_{K} X$, and the Abel-Jacobi map AJ : $S^{g} C \rightarrow \mathrm{Pic}_{C / K}^{g}$ (e.g., [Kle05, Def. 9.4.6, Rem. 9.3.9]).

Pulling back to $\bar{K}$, Brill-Noether theory provides the following two facts. First, the AbelJacobi map $\overline{\mathrm{AJ}}: S^{g} \bar{C} \rightarrow \operatorname{Pic} \frac{g}{\bar{C}} / \bar{K}$ is proper and birational, and the image of the exceptional locus is a set of dimension $g-2$ (this set is identified with the Serre dual of the Brill-Noether locus $\left.W_{g-2}(\bar{C})\right)$. Second, denoting by $\bar{p}_{1}, \bar{p}_{0}$ the $\bar{K}$-points associated to $p_{1}, p_{0}$, then if $\bar{p}_{2}, \ldots, \bar{p}_{g} \in C(\bar{K})$ are sufficiently general (and therefore can be taken to be defined over a finite separable extension of $L)$, the images $\bar{t}_{1}$ and $\bar{t}_{0}$ of $\bar{p}_{1}$ and $\bar{p}_{0}$ respectively under the map

$$
\begin{aligned}
& \bar{C} \longmapsto \operatorname{Pic}_{\bar{C} / \bar{K}}^{g} \\
& \bar{p} \longmapsto \bar{p}+\left(\bar{p}_{2}+\cdots+\bar{p}_{g}\right)
\end{aligned}
$$

avoid the exceptional locus of $\overline{\mathrm{AJ}}$. Now define a cycle $Z:=\left(\mathrm{AJ} \times \operatorname{id}_{X}\right)_{*} S^{g} Z^{\prime}$ on $\operatorname{Pic}_{C}^{g} \times_{K} X$. Then

$$
Z_{\bar{t}_{1}}-Z_{\bar{t}_{0}}=\left(Z_{\bar{p}_{1}}^{\prime}+Z_{\bar{p}_{2}}^{\prime}+\cdots+Z_{\bar{p}_{g}}^{\prime}\right)-\left(Z_{\bar{p}_{0}}^{\prime}+Z_{\bar{p}_{2}}^{\prime}+\cdots+Z_{\bar{p}_{g}}^{\prime}\right)=Z_{\bar{p}_{1}}^{\prime}-Z_{\bar{p}_{0}}^{\prime}=a_{\bar{K}} .
$$

Letting $L^{\prime} / L$ be the finite separable extension over which the $p_{i}$ are defined, we are done.

Remark B.3. It is not hard to write down examples where Weil's construction in the proof above necessitates a field extension. For instance, let $C / \mathbb{F}_{3}$ be the hyperelliptic curve of genus 2 with affine model $y^{2}=x^{5}-x^{2}-1$. Then $C\left(\mathbb{F}_{3}\right)=\{P, Q\}$, where $P=[0: 1: 0]$ and $Q=[2: 0: 1]$, and $S^{2}(C)\left(\mathbb{F}_{3}\right)=\{2[P], 2[Q],[P]+[Q]\}$. Since $h^{0}(2 P)=h^{0}(2 Q)=2>1$, there is no effective $\mathbb{F}_{3}$-rational divisor $D$ of degree $g-1=1$ such that $P+D$ and $Q+D$ are both Brill-Noether general.

\section{REFERENCES}

[ACMV17] Jeffrey D. Achter, Sebastian Casalaina-Martin, and Charles Vial, On descending cohomology geometrically, Compositio Mathematica 153 (2017), no. 7, 1446-1478.

[AK80] Allen B. Altman and Steven L. Kleiman, Compactifying the Picard scheme, Adv. in Math. 35 (1980), no. 1, 50-112. MR 555258

[Con07] Brian Conrad, Deligne's notes on Nagata compactifications, J. Ramanujan Math. Soc. 22 (2007), no. 3, 205-257. MR 2356346 
[CP16] François Charles and Bjorn Poonen, Bertini irreducibility theorems over finite fields, J. Amer. Math. Soc. 29 (2016), no. 1, 81-94. MR 3402695

[dJ96] A. J. de Jong, Smoothness, semi-stability and alterations, Inst. Hautes Études Sci. Publ. Math. (1996), no. 83, 51-93. MR 1423020

[Ful98] William Fulton, Intersection theory, second ed., Ergebnisse der Mathematik und ihrer Grenzgebiete. 3. Folge. A Series of Modern Surveys in Mathematics [Results in Mathematics and Related Areas. 3rd Series. A Series of Modern Surveys in Mathematics], vol. 2, Springer-Verlag, Berlin, 1998. MR 1644323 (99d:14003)

[Har73] Robin Hartshorne, Equivalence relations on algebraic cycles and subvarieties of small codimension, Algebraic geometry (Proc. Sympos. Pure Math., Vol. 29, Humboldt State Univ., Arcata, Calif., 1974), Amer. Math. Soc., Providence, R.I., 1973, pp. 129-164. MR 0369359

[Jan00] Uwe Jannsen, Equivalence relations on algebraic cycles, The arithmetic and geometry of algebraic cycles (Banff, AB, 1998), NATO Sci. Ser. C Math. Phys. Sci., vol. 548, Kluwer Acad. Publ., Dordrecht, 2000, pp. 225-260. MR 1744947

[Jou83] Jean-Pierre Jouanolou, Théorèmes de Bertini et applications, Progress in Mathematics, vol. 42, Birkhäuser Boston, Inc., Boston, MA, 1983. MR 725671

[Kle68] S. L. Kleiman, Algebraic cycles and the Weil conjectures, Dix exposés sur la cohomologie des schémas, North-Holland, Amsterdam; Masson, Paris, 1968, pp. 359-386. MR 0292838 (45 \#1920)

[Kle05] _ The Picard scheme, Fundamental Algebraic Geometry, Math. Surveys Monogr., vol. 123, Amer. Math. Soc., Providence, RI, 2005, pp. 235-321. MR 2223410

[Kol96] János Kollár, Rational curves on algebraic varieties, Ergebnisse der Mathematik und ihrer Grenzgebiete. 3. Folge. A Series of Modern Surveys in Mathematics [Results in Mathematics and Related Areas. 3rd Series. A Series of Modern Surveys in Mathematics], vol. 32, Springer-Verlag, Berlin, 1996. MR 1440180

[Lan59] Serge Lang, Abelian varieties, Interscience Tracts in Pure and Applied Mathematics. No. 7, Interscience Publishers, Inc., New York; Interscience Publishers Ltd., London, 1959. MR 0106225 (21 \#4959)

[Mum70] David Mumford, Abelian varieties, Tata Institute of Fundamental Research Studies in Mathematics, No. 5, Published for the Tata Institute of Fundamental Research, Bombay; Oxford University Press, London, 1970. MR 0282985 (44 \#219)

[Mur85] J. P. Murre, Applications of algebraic K-theory to the theory of algebraic cycles, Algebraic geometry, Sitges (Barcelona), 1983, Lecture Notes in Math., vol. 1124, Springer, Berlin, 1985, pp. 216-261. MR 805336 (87a:14006)

[Poo04] Bjorn Poonen, Bertini theorems over finite fields, Ann. of Math. (2) 160 (2004), no. 3, $1099-1127$. MR 2144974 (2006a:14035)

[Sam60] Pierre Samuel, Relations d'équivalence en géométrie algébrique, Proc. Internat. Congress Math. 1958, Cambridge Univ. Press, New York, 1960, pp. 470-487. MR 0116010

[Sch09] Stefan Schröer, On genus change in algebraic curves over imperfect fields, Proc. Amer. Math. Soc. 137 (2009), no. 4, 1239-1243. MR 2465645

[Sta17] The Stacks Project Authors, Stacks Project, http://stacks.math.columbia.edu, 2017.

[Wei54] André Weil, Sur les critères d'équivalence en géométrie algébrique, Math. Ann. 128 (1954), 95-127. MR $0065219(16,398 \mathrm{e})$

Colorado State University, Department of Mathematics, Fort Collins, CO 80523, USA

E-mail address: j.achter@colostate.edu

University of Colorado, Department of Mathematics, Boulder, CO 80309, USA

E-mail address: casa@math.colorado.edu

Universität Bielefeld, Fakultät für Mathematik, 33501 Bielefeld, Germany

E-mail address: vial@math.uni-bielefeld.de 